\title{
Permeability of polydisperse solid foams
}

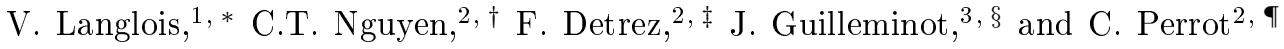 \\ ${ }^{1}$ Navier, Univ Gustave Eiffel, Ecole des Ponts, CNRS, F-77454 Marne-la-Vallée, France \\ ${ }^{2} M S M E$, Univ Gustave Eiffel, CNRS UMR 8208, \\ Univ Paris Est Creteil,F-774454 Marne-la-Vallée, France \\ ${ }^{3}$ Department of Civil and Environmental Engineering, \\ Duke University, Durham, North Carolina 27708, USA
}

\begin{abstract}
The effect of polydispersity on foam permeability is investigated by numerical simulations. Foam structures are first generated by Laguerre tessellations via the Neper software, and relaxed to minimize the surface energy via the Surface Evolver software. The fluid flow and permeability are then calculated by means of pore-network simulations, by considering either fully open-cell foams or foams with randomly-selected closed windows. Different configurations of window aperture are used, including identical window aperture size, identical window aperture ratio, or random window aperture ratio. The main results are obtained for the case of foams having identical and uniform window aperture ratios. For such foams and at constant mean pore size, foam permeability is found to strongly increase with the polydispersity degree. The numerical results are employed to discuss the validity of the mean pressure field assumption used to calculate the foam permeability, the effect of small pores, and the definition of an equivalent Kelvin foam size. We show that as long as the fluctuations of the window aperture ratio remain low, foam permeability can be estimated by using the mean pressure field hypothesis. The weak effect of small pores on permeability is related to their small contribution to the overall fluid volume fraction. Finally, various estimations of the equivalent Kelvin foam size based on pore-size distribution are proposed.
\end{abstract}

NOTICE: This is the authors' version of a paper published in Physical Review E [Langlois et al.(2022), Permeability of polydisperse solid foams, Phys. Rev. E, 105, 015101, doi: 10.1103/PhysRevE.105.015101].

\section{INTRODUCTION}

Liquid foam microstructure is made of membranes (also called films for liquid foams), ligaments or Plateau's borders (junction of three membranes), and vertices or nodes (junction of four ligaments). Solid foam microstructures inherit some properties of the original liquid foam microstructure (Fig. 1): in particular, the poresize distribution and pore position in the solid foam are similar to the bubble size distribution and bubble position in the liquid foam (if coalescence and ripening stay limited). Whereas membranes are necessary closed in liquid foam, they can be open or totally absent in solid foam [Fig. 1 (b)], hence allowing for the foam pores to be connected through windows [the term "window" will refer to the surface where a membrane is located before its possible opening, Fig. 11(b)]. As soon as the fraction of open membrane exceeds a certain amount, called the percolation threshold, a fluid flow through the solid foam is possible. Therefore, these materials can be used in various applications, such as heat-exchanger, catalyser, filter, and acoustic insulator [1. For such applications, the permeability is a key parameter to control and to ensure the efficiency of foam material. For example, in the context of sound absorption of monodisperse open-cell

\footnotetext{
* vincent.langlois@univ-eiffel.fr

$\dagger$ cong-truc.nguyen@univ-eiffel.fr

$\ddagger$ fabrice.detrez@univ-eiffel.fr

$\S$ johann.guilleminot@duke.edu

ฯ camille.perrot@univ-eiffel.tr
}
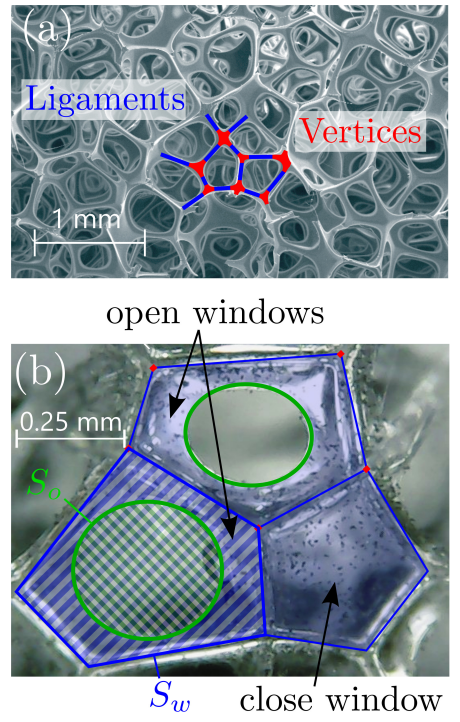

Figure 1. Microscopy images revealing the microstructure of solid foam: (a) ligaments (or Plateau borders, struts) and vertices, (b) open or closed windows (toner particles are spread over the foam sample to highlight the membrane surfaces).

foam, it was shown that modeling transport properties such as permeability is a key step to find the optimal microstructures defined by the pore size and the porosity [2, 3]. However, foams can have various degrees of polydispersity [4, 5] and/or various membrane contents [6, 7. Therefore, polydispersity and membrane content can be taken into account to search for new optimal foam microstructures. 
Different works have focused on the effects of morphological parameters - pore or strut diameter, porosity, specific surface area, window aperture size, etc. - on the permeability of monodisperse open-cell foams [1, 8, 9]. The effects of partially or fully closed membranes separating neighbor pores on foam permeability have recently been investigated [10].

Different approaches for modeling foam permeability can be found in the literature. A first approach is based on the so-called Carman-Kozeny equation [11, 12]: $K=C\left[\phi^{3} /(1-\phi)^{2}\right] d_{c}^{2}$ where $d_{c}$ is the inverse of the specific surface area $a_{c}$ (pore surface/solid volume), $\phi$ is the porosity, and $C$ is a dimensionless constant depending on the microstructure geometry. This equation was introduced to estimate the permeability of granular material. In the case of foam permeability, some alternative choices for the characteristic micro-structure length $d_{c}$ have been proposed in the literature, such as the pore size or the hydraulic radius $d_{h}=4 \phi / a_{c}$ (see Ref. [9] for a recent review). As $C$ depends on the microstructure geometry, these approaches cannot give a full analytical formulation and require numerical calculations to elucidate the relationship between $C$ and the microstructure geometry.

A second approach, especially relevant for foams having membranes [10] or for porous media having small constrictions connecting large pores [1, 13], was suggested by Despois and Mortensen [1. In this approach, the fluid flow passing through constrictions is supposed to be governed by the difference of fluid pressures between interconnected pores. The relationship between fluid flow and pressure drop used by Despois and Mortensen is due to Sampson [14. For monodisperse foam, the foam permeability can be fully derived by considering the symmetries of the periodic unit cell in the case of ordered foams [10], or by considering a mean pore in the case of disordered foams 13. This approach gives a very accurate estimation of foam permeability in a large range of constriction sizes. Moreover, this approach is similar to the porenetwork approach introduced by Fatt [15] to study the permeability of a network of tubes. The pore-network approach is also very useful to study the permeability of percolating porous media [16] and can be easily implemented in numerical calculations with low computational costs compared to other numerical methods (finite element, finite volume, boundary element). If the permeability of monodisperse and ordered foam has been extensively studied, then the permeability of polydisperse and disordered foams has received much less attention to date.

Due to the natural polydispersity of soil, the effects of polydispersity on the permeability of granular material have been studied in the past. Different equations for permeability can be found in the literature. For example, the Hazen formula [17, cited in numerous geotechnical textbooks [18-20, links the permeability $K$ to an effective size $D_{10}: K=C_{\text {Hazen }} D_{10}^{2}$ where $C_{\text {Hazen }}$ is an empirical constant, and $D_{10}$ is the grain size for which the mass of particles smaller than $D_{10}$ is equal to ten percent of the total mass of particles. However, Hazen formula is not very accurate to predict the permeability of granular material [21], and formulations derived from the CarmanKozeny formula give better predictions. Carrier [21] proposed the expression $d_{\mathrm{eff}}=\left(\sum_{i} x_{i} / d_{i}\right)^{-1}$ for the effective grain size to be used in the Carman-Kozeny formula instead of $d_{c}$; where $x_{i}$ is the mass fraction of particles having a size $d_{i}$. These formulas suggest that the sizes, $D_{10}$ and $d_{\text {eff }}$, may be interpreted as the grain size of an equivalent monodisperse granular media for the permeability. With a similar approach, formulas for the permeability of polydisperse foams involving the pore size of an equivalent monodisperse foam could perhaps be derived.

In this paper, pore-network simulations are used to study the permeability of random polydisperse foams with various membrane contents in the case of high porosity (corresponding to the "dry foam limit" in liquid foam, i.e, ligaments are lines, vertices are points and porosity $\phi$ is equal to 1$)$. In the first section, we detail the porenetwork method used for the calculation of permeability and the procedure used to generate numerical foam microstructures. Results concerning polydispersity degree and morphological parameters are presented in this section. The following section presents the results of porenetwork simulations performed on both fully open cell foam samples and foam structure having a fraction of closed membranes. The last section is devoted to the discussion of various points regarding the permeability of open cell foam samples: (i) the estimation of the permeability by assuming a mean pressure field, (ii) the effect of small pores and (iii) the estimate of the size of the equivalent monodisperse foam for the permeability, i.e. the pore size of a monodisperse Kelvin foam having the same permeability as the polydisperse foam sample.

\section{METHODS}

\section{A. Pore-network model for permeability}

Because most of the pressure drop is located in the vicinity of the membrane aperture, a simplified calculation of the fluid flow based on the pore-network hypothesis is possible [10]. In this framework, a value of fluid pressure is associated with each pore and the flow between two connected pores is based on a local fluid flow conductance [Figs. 2(a) and (b)]. Therefore, in the pore-network model, the details of local velocity and pressure fluctuations within a single pore are not described in detail. In the case of circular aperture of radius $r_{o, i}=d_{o, i} / 2$, the volume fluid flow rate $q_{i}$ passing through the window aperture $i$ depends on the pressure difference $\left(P_{i_{2}}-P_{i_{1}}\right)$ and on a local fluid flow conductance $G_{i}$, as established by Sampson [14]:

$$
q_{i_{2} \rightarrow i_{1}}=G_{i}\left(P_{i_{2}}-P_{i_{1}}\right), \quad \text { with } \quad G_{i}=\frac{r_{o, i}^{3}}{3 \mu}
$$




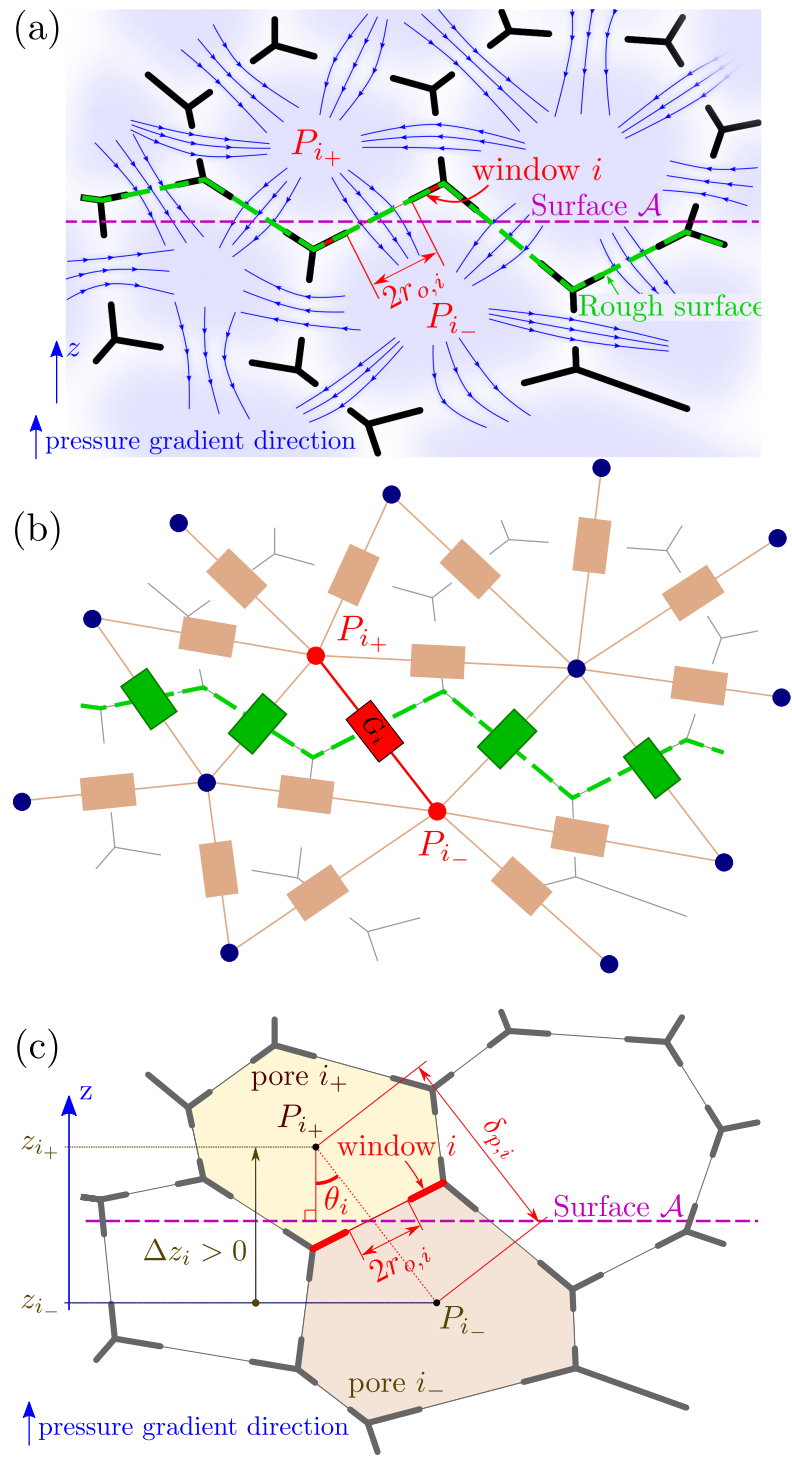

Figure 2. (a) Flow pattern and (b) its equivalent porenetwork scheme. (c) Definition of geometrical parameter involved in permeability calculation. The plane cross-section $\mathcal{A}$ of area $A$ divides the sample into two parts. If two pores, $i_{-}$ and $i_{+}$, associated with a window are located from either side of the cross-section, then the window is located on the rough surface.

where $\mu$ is the dynamic viscosity of the fluid.

The pore-network model describes the foam pore-space as a network of fluid flow conductances [Fig. 2(b)]. Only periodic networks are considered. The total number of elements within the elementary structure will be denoted $N_{p}$ for pores and $N_{w}$ for windows. Assuming that the flow of the incompressible fluid is steady, the sum of currents flowing towards or away from the pore $n$ is equal to zero (similarly to Kirchhoff's junction rule): $\sum_{m} G_{m n}\left(P_{m}-P_{n}\right)=0$, where $G_{m n}$ is equal to the local conductance associated with the window shared by the pores $n$ and $m$ (and equal to 0 if pores are not connected). A macroscopic pressure gradient is imposed by applying a macroscopic pressure difference $\Delta P_{\text {macro }}$ between upper and lower boundaries. When the pores are connected from top to bottom of the sample, the pressure in each pore can be calculated from the following matrix form equation:

$$
[G][P]=[F],
$$

where

- $[P]$ is the pressure vector for all pores;

- $[G]$ is the matrix of local conductances: $-\sum_{n} G_{m n}$ on the diagonal and $G_{m n}$ elsewhere;

- $[F]$ is a forcing vector containing zeros except for the components associated with the pores located on the upper and lower limits of the sample (the upper pores have lower pores as neighbors by periodicity and vice versa). For a boundary pore $m$, $F_{m}=\sum_{n, \text { lower }} G_{m n} \Delta P_{\text {macro }}$ if $m$ corresponds to an upper pore, while $F_{m}=-\sum_{n \text {,upper }} G_{m n} \Delta P_{\text {macro }}$ if the pore belongs to the lower boundary.

From the knowledge of pore pressures, the global flow rate $Q_{z}$ is equal to the sum of the local flow rates $q_{i}$ passing through the windows covering the rough surface associated with a cross-section $\mathcal{A}(z)$ of area $A$ [see Fig. 2(a) for a definition of the "rough surface"] :

$$
Q_{z}=\sum_{i=1}^{N_{w \mathcal{A}}} q_{i}=\sum_{i=1}^{N_{w \mathcal{A}}} G_{i} \Delta P_{i},
$$

where $\Delta P_{i}=P_{i_{+}}-P_{i_{-}}$[where $i_{+}$and $i_{-}$refers to the pores associated with the window " $i$ " with " $i_{+}$" located above the cross-section and " $i_{-}$" below, see Fig. 2 2c)].

As the fluid is considered incompressible, the global flow rate $Q_{z}$ is the same for any rough surface, or any continuous surface of windows joining lateral faces and respecting the lateral periodicity. In our calculation, we use the upper limit of the samples: the pore $i_{-}$is an upper pore and the pore $i_{+}$is a lower pore rebuilt by periodicity.

Finally, the permeability $K_{z}$ is calculated as follows :

$$
K_{z}=\mu \frac{Q_{z}}{A} \frac{1}{\lambda},
$$

where $\lambda=\frac{\Delta P}{H}$ and $H$ is the length of the sample in the direction of the macroscopic pressure gradient (equal to the spatial period in periodic structures). Note that for isotropic porous media: $K_{x}=K_{y}=K_{z}$.

From Eqs.(2)-(4), it appears that the macroscopic permeability $K$ depends on local conductances $G_{i}$ through the determination of the pressure field [Eq. (2)] and the global flow rate $Q_{z}$ [Eq. (3)]. It should be noticed that the effect of local conductances on the global flow rate is direct, whereas the effect on the pressure field is of the second order compared to that of the macroscopic pressure difference $\Delta P_{\text {macro }}$. For example, consider the case 
where the same coefficient $\alpha$ is applied to all local conductances (that is, $G_{i, 1}=\alpha G_{i, 0}$ ). From Eq. (2) and the expression of the forcing vector $[F]$, it appears that the local pressures are not modified $\left(P_{n, 1}=P_{n, 0}\right)$, while the coefficient $\alpha$ applies to both the total flow rate and the permeability $\left(K_{1}=\alpha K_{0}\right)$.

The pore-network model with Sampson's law has been successfully validated by comparing its predictions to numerical calculations on Kelvin structures and to experimental measurements performed on monodisperse foams [10, 13, 22. Pore-network models are usually considered to describe the conductivity through a network of tubes. In such a case, the local conductance is given by the Poiseuille law [23, 24]: $G_{\text {tube }}=\pi R_{t, i}^{4} /\left(8 \mu l_{t, i}\right)$, where $R_{t, i}$ and $l_{t, i}$ are the inner radii and the length of the tube $i$, respectively. In such a model, the distance between the connected pores corresponds to the length of the tubes. The pressure drop is uniformly distributed over the length of the tube, whereas in foams, the pressure drop is located near the membrane aperture. In a foam structure, the distances between pores and the positions of pores are not explicitly present in the porenetwork model. The input geometrical parameters of the pore-network model are the sizes of the membrane apertures and the structure of the pore network. This structure is specifically defined by knowing: (i) the indices of the pores associated with each window ; (ii) if a window is inside the periodic structure or located on its boundary ; (iii) which pore is inside or outside the periodic structure for a boundary window. Note that the connectivity of the pores in the network can be represented by a square matrix, called the adjacency matrix and denoted by $a_{i j}$, which is defined as follows [25]: $a_{i j}=1$ if the pores, $i$ and $j$, are neighbors, and 0 otherwise.

In Appendix A we present two alternative methods to calculate the permeability. The first approach follows from the definition of the permeability [Eq. (4)], while the second is based on energetic consideration. Moreover, we introduce two macroscopic parameters on which permeability can depend, namely the volume density of pores, given by $\rho_{p}=N_{p} / V$, and the volume density of windows, $\rho_{w}=N_{w} / V$ with $V=A H$, the volume of the elementary structure. Note that the inverse of the cube root of the pore density is a good estimator of the mean distance between pores $\left\langle\delta_{p}\right\rangle$. These methods lead to the following expressions for the permeability:

$$
\begin{aligned}
K_{\mathrm{av}} & =\frac{\mu \rho_{w}}{\lambda}\langle G \Delta P \Delta z\rangle_{w}, \\
K_{e} & =\frac{\mu \rho_{w}}{\lambda^{2}}\left\langle G|\Delta P|^{2}\right\rangle_{w},
\end{aligned}
$$

where \langle\rangle$_{w}=\frac{1}{N_{w}} \sum_{i \in\{w\}}$ is the average over the windows. Our pore network simulations show that Eqs. (4), (5) and (6) lead to the same values of permeability. Because a term $\Delta z_{i}=z_{i_{+}}-z_{i_{-}}$appears in Eq. (5), the positions of the pores seem to have a direct effect on permeability. This result, however, is in contradiction with the previous discussion and the form of Eq. (6). To check that permeability does not depend on the exact positions of the pore centroids (with a fixed structure of the pore network), we performed calculations by adding a random term to the position of the pores, and obtained results identical to the ones without the perturbations. However, it is important to note that the spatial distribution of pores leading to $\Delta z_{i}$, affects the permeability by its effect on the structure of the pore network.

For any window $i$, the pressure drop $\Delta P_{i}$ is proportional to $\Delta P_{\text {macro }}$. Moreover, increasing the number of pores (or the pore density $\rho_{p}$ ) by a factor $2^{3}$ will increase by a factor 2 the number of pressure drops when the fluid flows through the pore network. Therefore, the pressure drop can be written as $\Delta P_{i}=\lambda \rho_{p}^{-1 / 3} \Delta \tilde{P}_{i}$. Similarly to the pressure field $P$, the normalized pressure $\tilde{P}$ depends on the structure of the network and the normalized local conductances $G_{i} /\langle G\rangle_{w}=r_{o, i}^{3} /\left\langle r_{o}^{3}\right\rangle_{w}$. However, unlike $P$, $\tilde{P}$ does not depend on the size of the elementary structure (while its size is larger than the size of the representative elementary volume). Combining these results with Eq. (6) yields to the following expression for the permeability:

$$
K=\frac{\rho_{w}}{\rho_{p}^{2 / 3}}\langle\mu G\rangle_{w} \mathcal{F},
$$

where $\mathcal{F}=\left\langle r_{o}^{3}(\Delta \tilde{P})^{2}\right\rangle_{w} /\left\langle r_{o}^{3}\right\rangle_{w}$ is a dimensionless function depending on the structure of the network and the normalized local conductances $G_{i} /\langle G\rangle_{w}=r_{o, i}^{3} /\left\langle r_{o}^{3}\right\rangle_{w}$. A similar reasoning can be used to derive the same kind of dimensionless function with Eq. (5), because $\Delta z_{i} / H$ is sensitive to the same size effects than the inverse of the number of pressure drops.

\section{B. Numerical microstructure of random polydisperse foams}

The process used to generate $3 \mathrm{D}$ periodic structures of random polydisperse foam is inspired by the work of Kraynik and co-authors [26-29]. It involves two steps: (1) filling space with Laguerre polyhedra and (2) relaxing the Laguerre structures to satisfy Plateau's laws and minimize surface area. The pore geometry evolves during the relaxation calculation: starting from polyhedra with flat faces and straight ligaments [Fig. 3(a)] to converge to pores with curved faces and ligaments [Fig. 3. (b)]. Note that the pore-size distribution remains unchanged during the relaxation process, while the sphericity is deeply affected by the curvatures of interfaces, leading to a mean sphericity $(\langle\Psi\rangle \approx 0.91)$ independent on the polydispersity degree (as shown previously by Kraynik et al. [27] and illustrated in Fig. 4 for our relaxed foam samples). In this work, each step is based on the use of a specific powerful and free software: Neper software for the generation of periodic and isotropic Laguerre structures [30] and Surface Evolver for the relaxation calculation [31. 
a
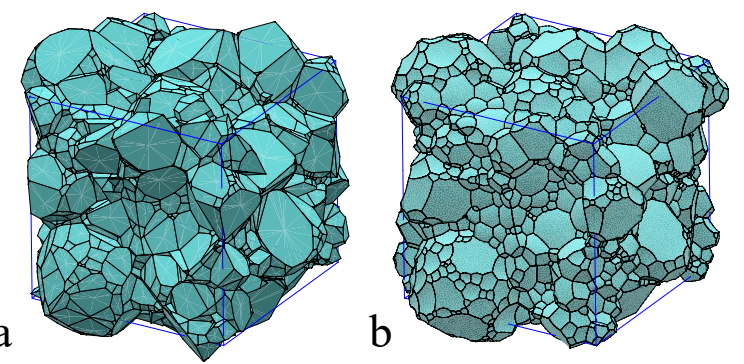

Figure 3. (a) 3D periodic Laguerre microstructure performed by Neper (path $1, C_{d}=0.95$ ), (b) the same microstructure after relaxation calculated by Surface Evolver.

In the rest of this section, we first introduce some common parameters to Laguerre structures and to the relaxed ones. Then, we give some details concerning both calculation steps and present some results related to structural parameters involved in pore-network model.

\section{Microstructural parameters}

We define two microstructural parameters characterizing the pore size and the pore shape (compared to a sphere). The pore size is defined by a sphere equivalent diameter $d_{p}$, which represents the diameter of a sphere having a volume equal to the pore volume $V_{p}$. The pore shape is measured by the sphericity $\Psi$, which is defined as the ratio of the surface area of a sphere having the same volume as the pore to the surface area $S_{p}$ of the pore: $\Psi=(36 \pi)^{1 / 3} V_{p}^{2 / 3} / S_{p}$. The pore sphericity takes a maximal value equal to one for a spherical pore. For example, the sphericity is equal to 0.67 for a regular tetrahedron, 0.81 for a cube and 0.91 for a Kelvin structure (truncated octahedra). Moreover, we define the face size (or window size) as the diameter $d_{w}$ of a circle having an area equal to the face area $S_{w}$ (see Fig. 1): $d_{w}=2 r_{w}=\left(4 S_{w} / \pi\right)^{0.5}$. Note that the face surface area takes into account the curvature of the membrane in the relaxed structures.

From the pore-size distribution, different pore size averages can be defined based on the weighting of the mean. The calculation of these averages follows the general formula:

$$
d_{m, n}=\left(\frac{\sum_{p} d_{p}^{m}}{\sum_{p} d_{p}^{n}}\right)^{1 /(m-n)} .
$$

In this paper, we will consider three different pore diameter averages: the number-weighted mean diameter $d_{1,0}=\left\langle d_{p}\right\rangle$ ( $\langle$.$\rangle denotes the number average), the vol-$ ume/surface mean (Sauter) diameter $d_{3,2}=\left\langle d_{p}^{3}\right\rangle /\left\langle d_{p}^{2}\right\rangle$, and the diameter of the sphere having a volume equal to the mean pore volume $\left\langle V_{p}\right\rangle, d_{3,0}=\left\langle d_{p}^{3}\right\rangle^{1 / 3}$. It is worth mentioning that the size $d_{3,0}$ is related to the pore density $\rho_{p}$ through the relationship $d_{3,0}=\left(\frac{\pi}{6 \phi} \rho_{p}\right)^{-1 / 3}$ (with $\phi=1$ in the "dry foam limit"), and that $d_{3,0} / d_{1,0} \approx$

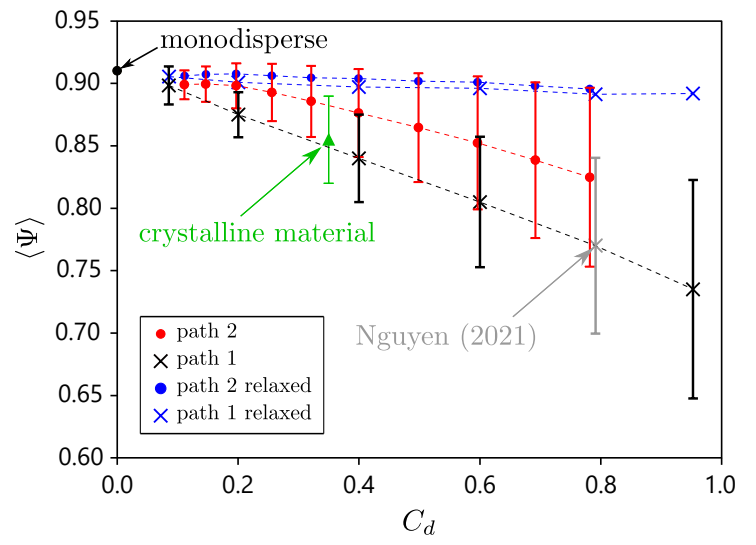

Figure 4. Mean sphericity $\langle\Psi\rangle$ against polydispersity parameter $C_{d}$. Error bars correspond to standard deviations. The point "monodisperse" corresponds to the sphericity of Kelvin or Weaire-Phelan structure. The point "crystalline material" corresponds to the sphericity parameters found in polycrystalline materials as reported in Ref. [32.

$1.0119 C_{d}^{2}-0.0107 C_{d}+1$ when the pore size follows a log-normal distribution.

To measure the polydispersity degree, we used the normalized standard deviation of pore sizes $C_{d}$ defined as:

$$
C_{d}=\frac{\sigma_{d_{p}}}{d_{1,0}}
$$

where $\sigma_{d_{p}}$ is the number-weighted standard deviation of pore sizes.

Similarly, the pore sphericity is characterized by the number-weighted mean $\langle\Psi\rangle$ and the standard deviation $\sigma_{\Psi}$ value (or the pore sphericity variation coefficient $\left.C_{\Psi}=\sigma_{\Psi} /\langle\Psi\rangle\right)$.

\section{Polydisperse Laguerre structures}

Neper software uses the distributions of pore size $d_{p}$ and sphericity $\Psi$ as inputs (i.e., the probability distributions and the associated hyperparameters - typically a mean and a standard deviation - must be specified). Concerning the probability distribution, we use lognormal distributions for the pore size and the variable $(1-\Psi)$, so that highly polydisperse microstructures can be obtained. Different Laguerre structures were generated by changing the polydispersity parameter $C_{d}$ with the mean pore size $\left\langle d_{p}\right\rangle=d_{1,0}$ set to 1.0 .

We have considered two ways to modify $\langle\Psi\rangle$ and $C_{\Psi}$ as the polydispersity parameter $C_{d}$ changes: (1) in the first case ("path 1"), we considered known structures of foams, (2) in the second case ("path 2"), we consider Laguerre microstructures maximizing $\langle\Psi\rangle$ for each polydispersity parameter $C_{d}$. In the first case ("path 1"), we relied on known structures of foam samples: monodisperse foams and a highly polydisperse polyurethane foam sample (characterized by x-ray microtomography, see Ref. [5]). 

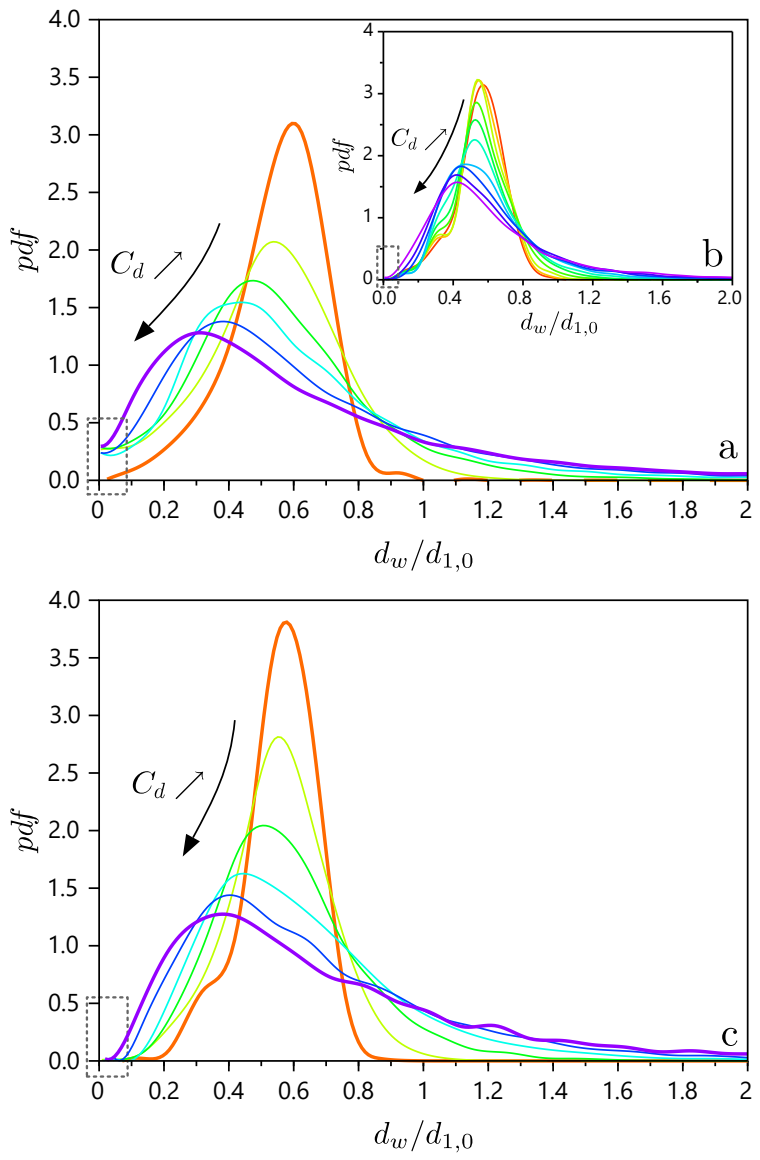

Figure 5. Window size distributions for Laguerre and relaxed microstructures: (a) Laguerre "path 1", (b) Laguerre "path 2", and (c) relaxed "path 1". Note that dotted rectangles are added to graphs in the vicinity of their origin to highlight that the Laguerre "path 1" condition generates polyhedra with small faces unlike the other conditions.

The structure of monodisperse foam samples can be approximated by ordered monodisperse structures such as the Kelvin structure and that of Weaire-Phelan [33, 34]. For these two ordered monodisperse structures, we have $C_{d}=0,\langle\Psi\rangle \approx 0.91$ and $C_{\Psi}=0$. Similar values for $\langle\Psi\rangle$ are reported for random monodisperse foam in Ref. [35. (note that the sphericity $\Psi$ corresponds to the inverse of the parameter $\beta$ used in Ref. [35]). The microstructure of our highly polydisperse polyurethane foam sample is characterized by $\langle\Psi\rangle=0.77, \sigma_{\Psi}=0.07$, and $C_{d}=0.79$. Therefore, for the first case, the pore sphericity $(\langle\Psi\rangle$ and $C_{\Psi}$ ) for different values of $C_{d}$ is linearly interpolated between the pore sphericity of monodisperse foam $\left(C_{d} \approx 0\right.$, $\left.\langle\Psi\rangle=0.91, C_{\Psi}=0\right)$ and the pore sphericity of the real polydisperse $\mathrm{PU}$ foam sample having $\left(C_{d}=0.79\right.$, $\left.\langle\Psi\rangle=0.77, C_{\Psi}=0.09\right)$. The evolution of sphericity parameters are plotted against $C_{d}$ for the simulations used to generate Laguerre tessellations in Fig. 4. Note that this path of pore sphericity evolution with $C_{d}$ encompasses a case observed on polycrystals for which the size distribution of crystals is governed by the minimization of the interfacial energy [32, 36]. Unfortunately, Laguerre tessellation is known to produce polyhedra having very small faces [26-28, 30]. Fig 5 (a) shows the distribution of face sizes $d_{w}$ and confirms that the number of very small faces rises as polydispersity increases.

(2) A second path was explored to limit the generation of polyhedra having small faces. The strategy is based on the observation that Laguerre microstructures maximizing $\langle\Psi\rangle$ for each polydispersity parameter $C_{d}$ do not exhibit the above limitation [Fig[5(b)].

For each configuration of input parameters $\left(C_{d},\langle\Psi\rangle\right.$, $C_{\Psi}$ ), five samples were generated with at least 1000 pores to ensure that the averages of the parameters of interest over the five samples did not depend on the number of pores (see details in Ref. [5]). The range of variation of the polydispersity degree $C_{d}$ is 0.09 to 0.95 for "path 1 " and 0.11 to 0.78 for "path 2".

\section{Relaxed microstructures}

The Surface Evolver is used to relax the Laguerre structures, i.e., to minimize the surface area (surface energy, see Appendix B for details) and to satisfy Plateau's laws. Therefore, a significant fraction of small faces (or short edges) shrink to zero area (or zero length). All edges and faces smaller than $10^{-3} d_{1,0}$ for edges and $10^{-4} d_{1,0}^{2}$ for faces were removed to produce topological transitions favorable to surface-area minimization. Consequently, the relaxation process modifies the windows size distribution as shown in Fig. 5 the large amount of small faces observed in a Laguerre structure [Fig. [5(a)] is not present in the relaxed structure [Fig. 5(c)]. The relaxation process is time consuming, and its duration depends on the structure of the initial Laguerre samples: to few hours for low $C_{d}$ up to few weeks for high $C_{d}$ (with Surface Evolver running on eight processors). Moreover, the microstructure does not evolve linearly with the computation time: numerous topological transitions occur in the beginning of the calculation, and as the microstructure relaxes, an additional topological transition requires more computation time.

\section{Structural parameters involved in pore-network model}

The pore-network model emphasizes different structural effects on permeability, namely (1) effects of the pore network structure and (2) local conductance effects.

Topological effects can be partly estimated via the number of neighboring pores $N_{v}$. It is an important parameter in the context of fluid flow through foam samples, particularly when they contain closed membranes [10. The mean number of neighboring pores $\left\langle N_{v}\right\rangle$ is an overall measure of the connectivity degree of pores and is useful to monitor the evolution of the pore network structure. The evolution of the distribution of $N_{v}$ with the polydispersity degree is given in Appendix B but global 
information can be found by using the mean number of neighbors $\left\langle N_{v}\right\rangle$ and the normalized standard deviation $C_{N_{v}}$ of the number of neighbors. The density of windows $\rho_{w}$ and the density of pores $\rho_{p}$ are both affected by the polydispersity and are related to the average number of neighbors $\left\langle N_{v}\right\rangle: \rho_{w}=\rho_{p}\left\langle N_{v}\right\rangle / 2$. Figure 6 shows the evolution of $\left\langle N_{v}\right\rangle$ and $C_{N_{v}}$ with the polydispersity degree, and compares our results to experimental results found in literature and numerical results determined by Kraynik et al. 27. from their simulations of random soap froth. As a general trend, the average number of neighbors $\left\langle N_{v}\right\rangle$ decreases as the polydispersity $C_{d}$ increases [Fig. 6(a)] ; and the average number of neighbors $\left\langle N_{v}\right\rangle$ decreases with the relaxation process. This effect is more pronounced in the case of "path 1" which has a large amount of small faces at the beginning of the relaxation process and consequently starts far from the relaxed state. Note that our results differ from Ref. [27] here. The observed discrepancies may be explained by the values for the cut-off thresholds in edge length and face area chosen for the relaxation process. In our simulations, we used very low threshold values, while Kraynik et al. chose larger values that lead to the removal of an important amount of connections between pores. The differences between numerical results and the fact that they do not reproduce experimental data can be understood by considering that there is no unique relationship between $\left\langle N_{v}\right\rangle$ and $C_{d}$ : $C_{d}$ essentially depends on the distribution of the pore size and $\left\langle N_{v}\right\rangle$ depends on the way the pores are arranged in space (and on the curvature of the interfaces). Concerning the normalized standard deviation $C_{N_{v}}$, which is a measure of the topological disorder [27, Figure 6.(b) shows that it increases with polydispersity. Moreover, our numerical results are quite similar to experimental results found in literature [5, 29].

Concerning the second structural effect, the local conductance depends on the window aperture size, which is always less than or equal to the window size: $G_{w}=$ $r_{o}^{3} / 3 \mu \leq r_{w}^{3} / 3 \mu=d_{w}^{3} / 24 \mu$. Since window aperture is left undefined at this stage, we focus on the effect of polydispersity on $\left\langle d_{w}^{3}\right\rangle$ below. As shown in Fig. $7\left\langle d_{w}^{3}\right\rangle / d_{1,0}^{3}$ significantly increases together with the polydispersity degree. The relationships between $\left\langle d_{w}^{3}\right\rangle / d_{1,0}^{3}$ and $C_{d}$ are similar for all foam structures except for Laguerre structures "path 1". At a constant mean pore size $d_{1,0}$, we finally get as a consequence that the mean local conductance must significantly increase with the foam polydispersity (if the aperture size $r_{o}$ evolves as $d_{w}$ ). In fact, the evolution of $\left\langle r_{w}^{3}\right\rangle_{w}$ with $C_{d}$ is similar to that of $d_{3,0}^{3}$ since the ratio $\left\langle r_{w}^{3}\right\rangle_{w} / d_{3,0}^{3}$ is almost constant in the whole range of $C_{d}:\left\langle r_{w}^{3}\right\rangle_{w} / d_{3,0}^{3}=0.0244 \pm 0.010$ for relaxed structures, and $0.0265 \pm 0.025$ for Laguerre structures.

Additional results concerning foam microstructure are given in Appendix B,
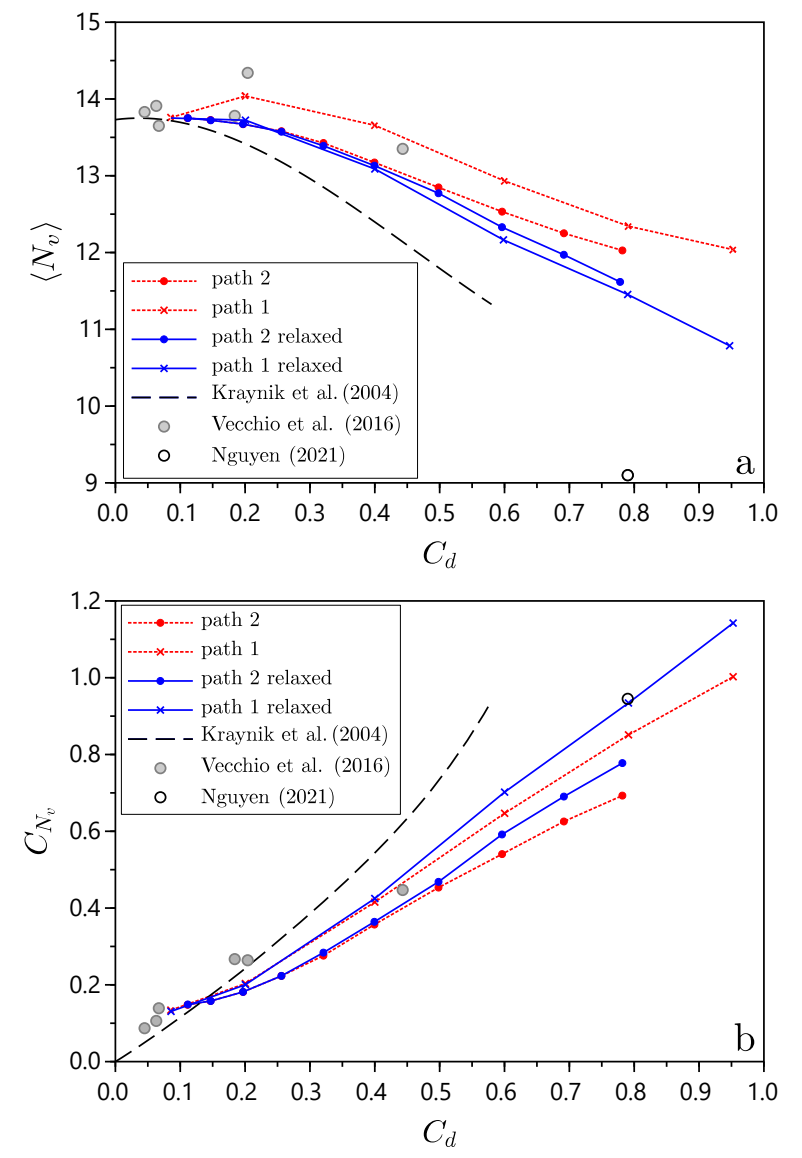

Figure 6. Number of neighboring pores $N_{v}$ : (a) mean value $\left\langle N_{v}\right\rangle$ and (b) normalized standard deviation $C_{N_{v}}$.

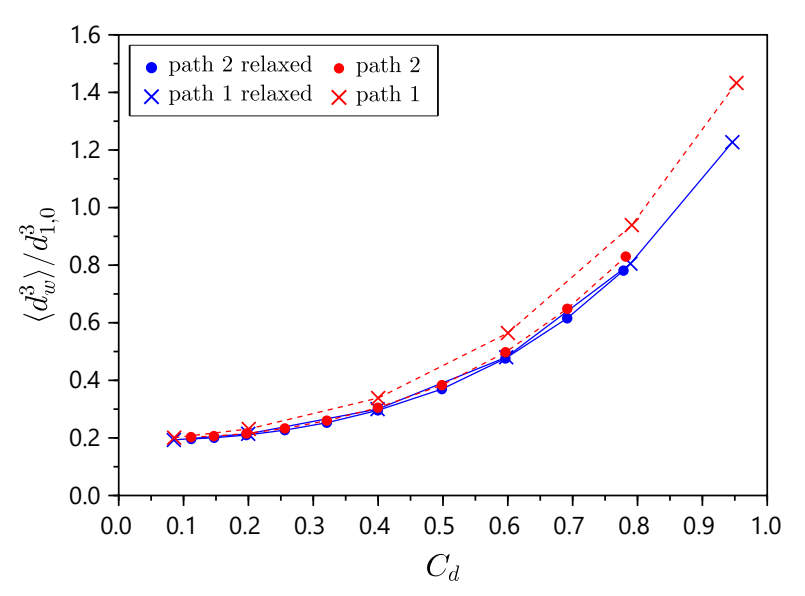

Figure 7. Normalized mean of the cubed window size $\left\langle d_{w}^{3}\right\rangle / d_{1,0}^{3}$ against $C_{d}$.

\section{PORE-NETWORK RESULTS}

\section{A. Permeability of polydisperse open-cell foam}

In this section, we pay attention to fully open-cell polydisperse foams (i.e., the membranes separating neighbor- 
ing pores in Laguerre or relaxed structure have holes). Three cases are specifically considered for the permeability calculations by pore-network simulations: (1) all windows are open with an identical aperture size $r_{o}$ proportional to the mean pore size $d_{1,0}: r_{o, i}=r_{o}$ regardless of the window $i$ and the polydispersity degree $C_{d}$ (we arbitrarily chose $r_{o}=3^{1 / 3} d_{1,0}$ which does not matter if the values of permeability are normalized by $\left.\mu G_{0} / d_{1,0}\right) ;(2)$ all windows have an identical aperture ratio $t_{o}\left(t_{o, i}=t_{o}\right.$ whatever the window $i$ and whatever the polydispersity degree $C_{d}$ ) where $t_{o}=\sqrt{S_{o} / S_{w}}=r_{o} / r_{w}$ with $S_{o}$ the area of the window aperture, $S_{w}$ the total area of the window, and $r_{w}=d_{w} / 2$ the equivalent window radius; (3) the aperture ratios of windows $t_{o, i}$ are drawn randomly, according to a uniform law on the interval $\left[0.5-\Delta t_{o}, 0.5+\Delta t_{o}\right]$, where $\Delta t_{o}$ varies between 0.01 and 0.49. Consequently, $\left\langle t_{o}\right\rangle=0.5$ and the standard deviation of $t_{o}$ is equal to $\Delta t_{o} / 3^{0.5}$. In the third case, calculations were repeated on 40 independent realizations and averaged.

The first case " $r_{o}$ cst" focuses on topological disorder because, at constant mean pore size $d_{1,0}$, all local conductances $G_{i}$ where equal to $G_{o}=\frac{1}{3 \mu} r_{o}^{3}$, regardless of the window and the polydispersity degree $C_{d}$. This case is addressed more for theoretical reasons than for practical purposes, because it would be correspond to cases where the aperture sizes would be lower than the smallest pores (leading to very low permeability material in highly polydisperse structures). The second case " $t_{o}$ cst" combines both topological disorder and window size fluctuations (two disorder sources which are correlated since coming from the foam morphology). As the window size fluctuates, the local conductances $G_{i}$ are different for each window: $G_{i}=t_{o}^{3} r_{w, i}^{3} /(3 \mu)$. This case is of practical interest because there are some experimental evidences suggesting that real foams tend to satisfy this hypothesis [7]. The third case " $t_{o}$ random" introduces a random variable to the aperture rate as expected for real foams. It emphasizes the effect of the window size fluctuations on local conductances: $G_{i}=t_{o, i}^{3} r_{w, i}^{3} /(3 \mu)$ where $t_{o, i}$ and $r_{w, i}$ are independent random variables.

Note that the cases, " $r_{o}$ cst" and " $t_{o}$ cst", were previously considered for the permeability calculation of ordered Kelvin foam in Refs. [10, 22]. In such foam structures, symmetries of the periodic unit cell simplifies the problem resolution and analytical formulas can be derived (in the "dry foam limit"):

$$
\begin{aligned}
& K=4\left(\frac{3}{\pi}\right)^{1 / 3} \frac{\mu G_{0}}{d_{1,0}}, \quad \text { for " } r_{o} \text { cst", } \\
& K=c_{K} t_{o}^{3} d_{1,0}^{2}, \quad \text { for " } t_{o} \text { cst", }
\end{aligned}
$$

where $c_{K} \approx 0.0283$.

The evolution of the permeability as a function of the polydispersity degree $C_{d}$ for the case of a constant aperture size (" $r_{o}$ cst") and a constant aperture ratio (" $t_{o}$ cst") is plotted in Fig. 8. Foam permeabilities of low polydispersity degree are well described by the formulas
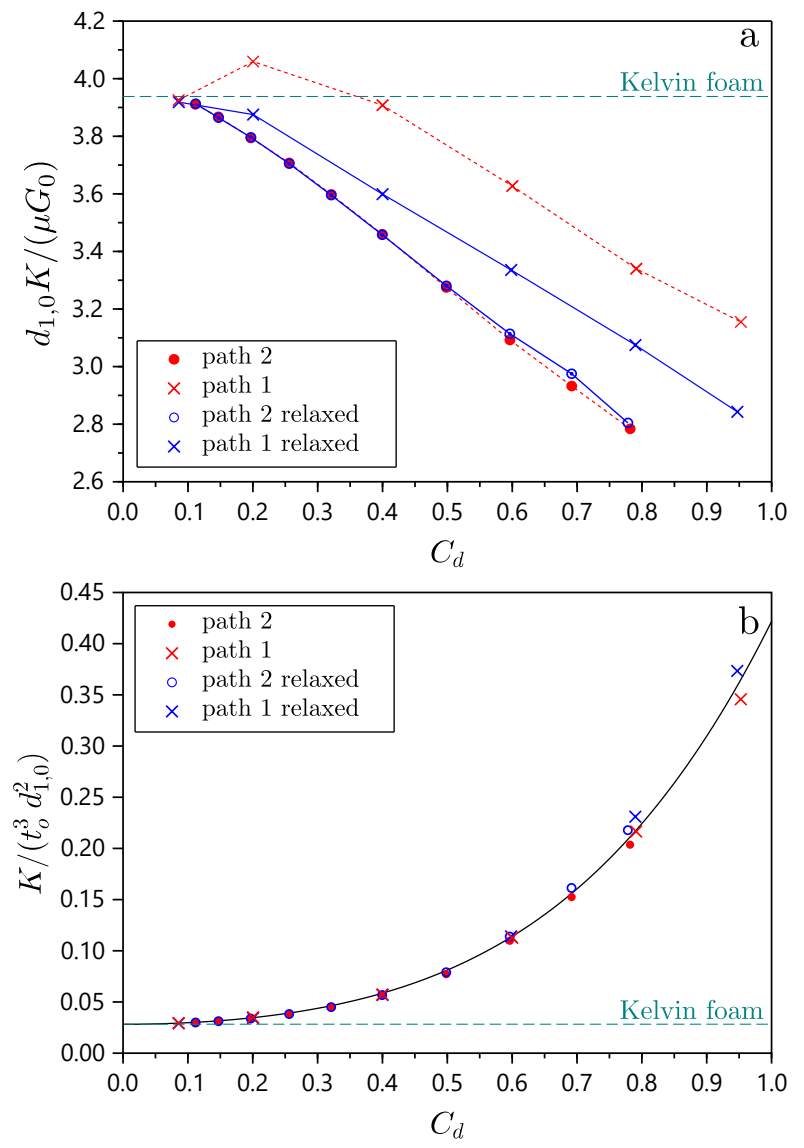

Figure 8. Permeability calculated by pore-network method for: (a) constant aperture size (" $r_{o}$ cst"), (b) constant aperture ratio (" $t_{o}$ cst"). In panel (b), the solid line is $0.0283(1+$ $\left.5.35 C_{d}^{2}+8.56 C_{d}^{4}\right)$.

obtained for Kelvin foam for both cases [Eqs. 110] and (11)]. The case of constant aperture size " $r_{o}$ cst" gives a decreasing trend of permeability with the polydisperse degree $C_{d}$ (at $d_{1,0}$ and $G_{o}$ constant), while the permeability increases strongly for the case of constant aperture ratio $t_{o}$ (at $d_{1,0}$ constant). We recall that the local conductances are constant in the case of constant aperture size while they increase with the polydispersity degree in the case of constant aperture ratio. The increase of permeability with polydispersity has been shown by Skibinski et al. 37] with permeability measurements on model open-cell foams without membrane. Besides, the fact that increasing the variation coefficient of window sizes increases the permeability was also found in Westhoff et al. 38. As shown in Sec. IIB different morphological parameters $\left(\left\langle N_{v}\right\rangle,\langle G\rangle_{w}, \rho_{p}, \rho_{w}\right.$, and $\left.C_{N_{v}}\right)$ evolve as the polydispersity degree changes. To distinguish their various contributions to the permeability, we introduced previously (Sec. II A) the dimensionless function $\mathcal{F}=K \rho_{p}^{2 / 3} /\left(\rho_{w}\langle\mu G\rangle_{w}\right)$. This function allows us to separate the effects of (1) both the relative fluctuations of local conductances and the structure evolution from (2) 

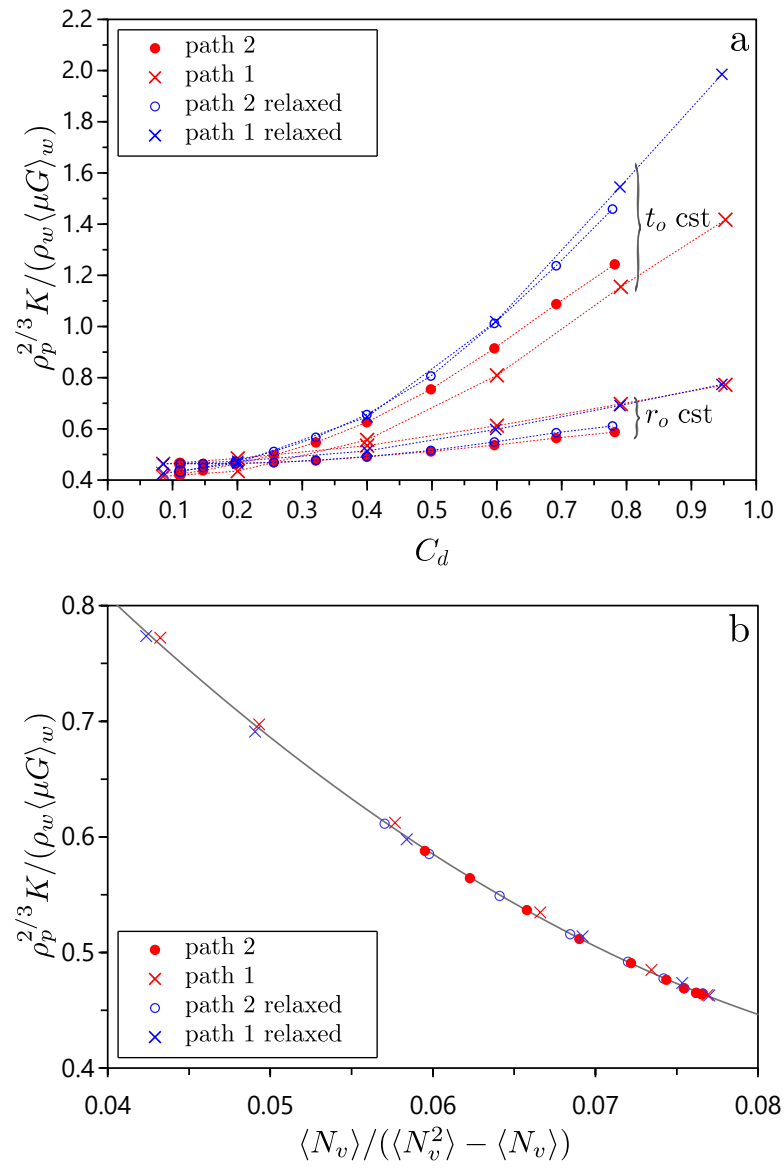

Figure 9. (a) Dimensionless function $\mathcal{F}=$ $K \rho_{p}^{2 / 3} /\left(\rho_{w}\langle\mu G\rangle_{w}\right)$ against $C_{d}$ [see Eq. $[7)$ ]. (b) $\mathcal{F}$ against $\left\langle N_{v}\right\rangle /\left(\left\langle N_{v}^{2}\right\rangle-\left\langle N_{v}\right\rangle\right)$ in the case of constant aperture size (" $r_{o}$ cst"). The solid line is $105 X^{2}-21.6 X+1.51$ with $X$ the abscissa. Note that $\frac{\rho_{p}^{2 / 3}}{\rho_{w}}=\left[\frac{4 \pi}{3}\right]^{1 / 3} \frac{d_{3,0}}{\left\langle N_{v}\right\rangle}$.

the effects of the evolution of the macroscopic (or mean) parameters $\left(\rho_{p}, \rho_{w},\left\langle N_{v}\right\rangle,\langle G\rangle_{w}\right)$. The permeability $K$ depends on the whole effects, whereas $\mathcal{F}$ depends only on the former (1). Figure 9(a) shows the evolution of $\mathcal{F}$ as a function of the polydispersity degree $C_{d}$. With this representation, the normalized permeability $\mathcal{F}$ increases with the polydisersity degree $C_{d}$ for both cases " $r_{o}$ cst" and " $t_{o}$ cst". The function $\mathcal{F}$ is especially interesting in the case of constant aperture size (" $r_{o}$ cst"), because it is expected that the function depends only on parameters describing the pore-network structure (such as $\left\langle N_{v}\right\rangle$ or $\left.C_{N_{v}}\right)$. In the next section, we will consider the parameter $\left\langle N_{v}\right\rangle /\left(\left\langle N_{v}^{2}\right\rangle-\left\langle N_{v}\right\rangle\right)$ to build an approximate expression for the percolation threshold. In Fig. 9 (b), we show that we obtain a master curve $\mathcal{F}\left(\left\langle N_{v}\right\rangle /\left(\left\langle N_{v}^{2}\right\rangle-\left\langle N_{v}\right\rangle\right)\right)$ with all data obtained for the case of constant aperture size (" $r_{o}$ cst").

The effect of a random variation of $t_{o}$ on the permeability is shown in Fig. 10 for structures having various polydispersity degree $C_{d}$. For all polydispersity de-

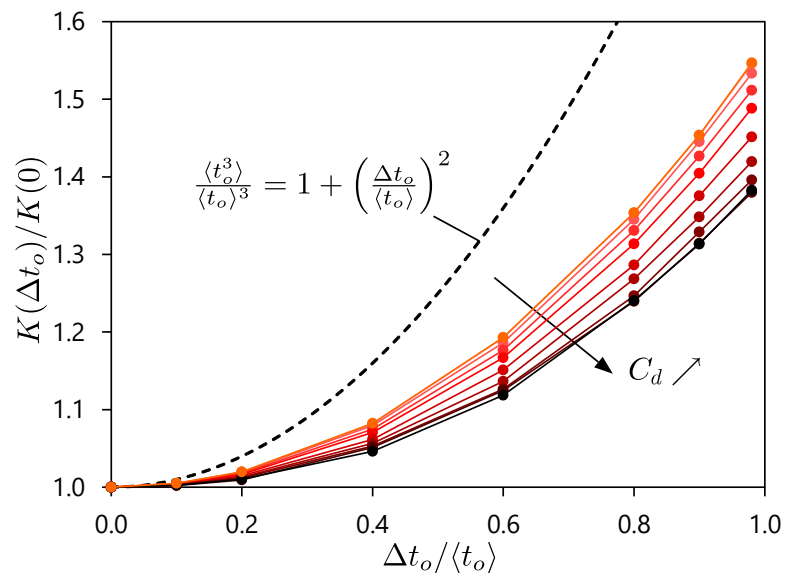

Figure 10. Permeability evolution against $\Delta t_{o} /\left\langle t_{o}\right\rangle$ for "path 2 " relaxed structure and the case of random aperture ratio (" $t_{o}$ random"). The same trends are observed with the "path 1 " condition and the unrelaxed structures. Note that $\left\langle t_{o}\right\rangle=$ 0.5 and the standard deviation of $t_{o}$ is $\Delta t_{o} / 3^{0.5}$ in our porenetwork calculations. $C_{d}$ varies from 0.11 to 0.78 .

grees, the permeability increases with $\Delta t_{o} /\left\langle t_{o}\right\rangle$, although the effect is more pronounced when the polydispersity is low. As for the case " $t_{o}$ cst", the increase of permeability with $\Delta t_{o} /\left\langle t_{o}\right\rangle$ is an effect of the increase of the mean local conductance. Indeed, as $t_{o}$ is randomly chosen, the mean local conductance $\langle G\rangle$ is proportional to $\left\langle t_{o}^{3} d_{w}^{3}\right\rangle=\left\langle t_{o}^{3}\right\rangle\left\langle d_{w}^{3}\right\rangle=\left\langle t_{o}\right\rangle^{3}\left(1+\left(\Delta t_{o} /\left\langle t_{o}\right\rangle\right)^{2}\right)\left\langle d_{w}^{3}\right\rangle$. If the permeability were simply deduced from mean local conductance, then one would expect the permeability to increase as $1+\left(\Delta t_{o} /\left\langle t_{o}\right\rangle\right)^{2}$ and regardless of polydispersity degree. Figure 10 shows that the increase is less important than the one predicted by this simple expression. Actually, the fact that the mean local conductance $\langle G\rangle$ is inappropriate to calculate the foam permeability is perfectly known for monodisperse and ordered foam as shown in Ref. [10. In this case, the foam permeability is deduced from an effective local conductance which can be estimated by a specific averaging method given by Kirkpatrick [16] and not by the arithmetic average. However, this averaging method, effective for topologically ordered structures ( $N_{v}$ constant), is not appropriate for polydisperse structures.

\section{B. Effect of closed windows}

In this section, we present results concerning the effect of non-perforated membranes that close windows and prevent flow between neighboring pores from occurring. For each foam sample, a fraction of windows is closed with a random spatial distribution over the foam sample. The remaining windows are open either with the same aperture size " $r_{o}$ cst" or with the same aperture ratio " $t_{o}$ cst". The fraction of open windows is denoted $x_{\mathrm{ow}}$. To reduce the effects of the sample size, our nu- 

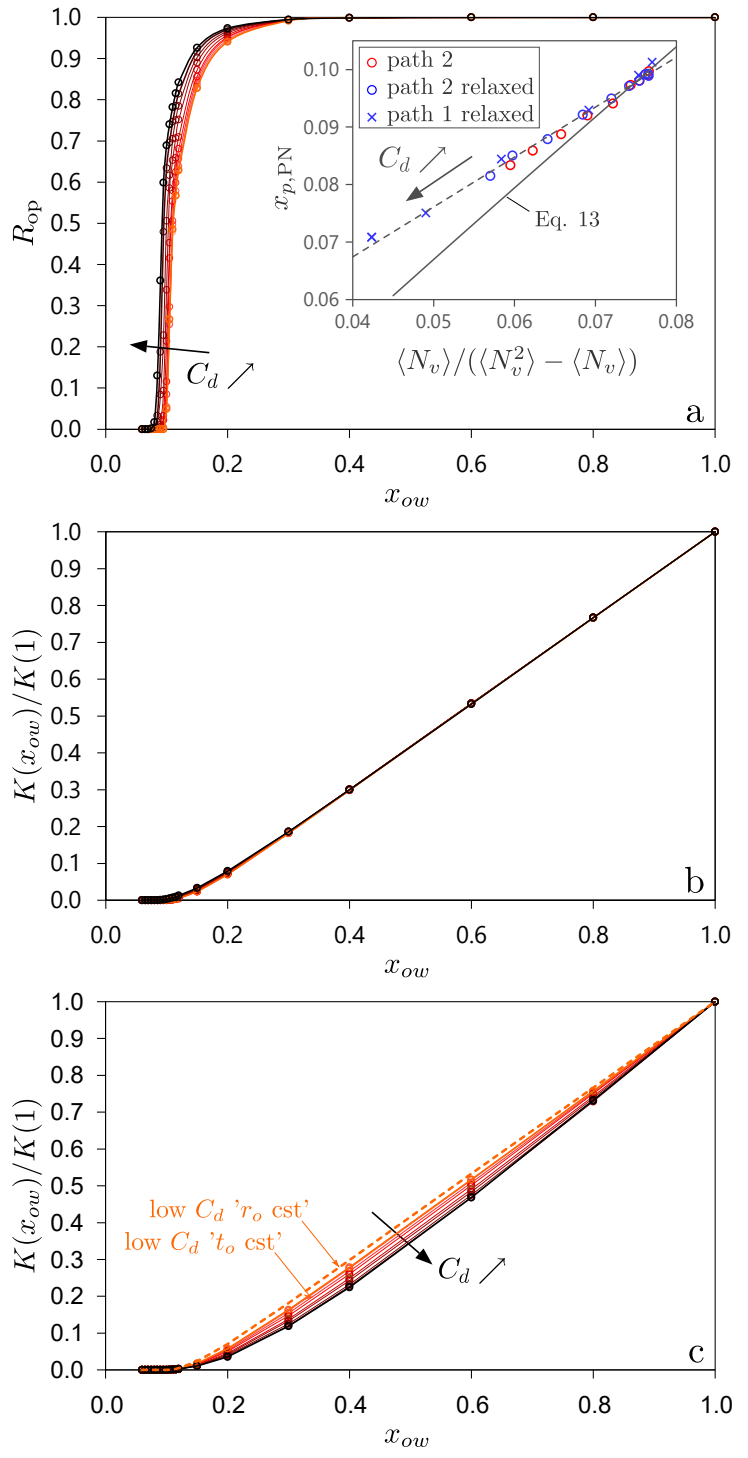

Figure 11. Effect of open window fraction $x_{\mathrm{ow}}$ and polydispersity degree $C_{d}$ on: (a) open porosity $R_{\text {op }}$ (defined as the ratio of the volume of the pores belonging to the percolating pore network to the volume of all the pores), and dimensionless permeability $K\left(x_{o}\right) / K(1)$ for (b) " $r_{o}$ cst" and (c) " $t_{o}$ cst". In panel (c), the dashed line corresponds to the case " $r_{o}$ cst" with low polydispersity and highlights the effect of the local conductance dispersion on the curve shape. Inset in panel (a) shows the percolation threshold calculated from Eq. 13) (full line) and the one estimated from pore-network results, $\alpha_{p}$,PN against the parameter $\left\langle N_{v}\right\rangle /\left(\left\langle N_{v}^{2}\right\rangle-\left\langle N_{v}\right\rangle\right)$. And, the dashed line is $0.865 X+0.033$ with $X=$ the abscissa.

merical structure are duplicated once in each direction before performing our pore-network simulations. Calculations were repeated on 80 independent realizations and averaged.

As shown in Ref. [10] for ordered monodisperse structures, foams containing both open and closed windows can be subjected to percolation phenomena. Fig. 11(a) shows that the pore space is closed (zero open poros-

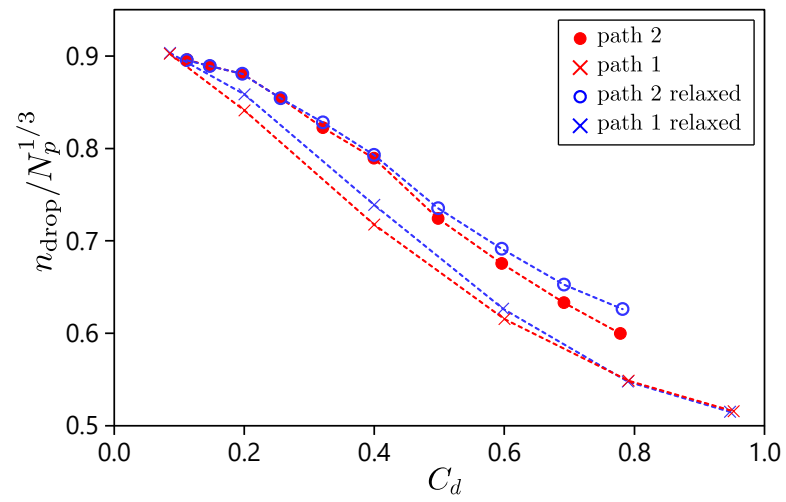

Figure 12. Mean number of drops $n_{\text {drop }}$ between pores and their translated counterpart against $C_{d}$. Note that $n_{\text {drop }}$ is normalized by $N_{p}^{1 / 3}$ to correct the size effect.

ity) until the fraction of open windows exceeds a value $x_{p}$, called the percolation threshold. For the foam structures considered in this work, the percolation threshold is around 0.1 and slightly decreases as the polydispersity degree increases (see Fig. 11.a) and the corresponding inset). This effect of polydispersity on the percolation threshold could be interpreted as the effect of large pores producing shortcuts in the random walk through the pore-network. Shortcuts then reduce the number of steps required to cross the sample. We have calculated the minimal number of pressure drops (or the minimal number of steps from pore to pore) required to connect each pore to its one-period-translated counterpart. This calculation is performed by using the property of the adjacency matrix (the term $i j$ of the $k$ th power of $a_{i j}$ is non zero if a path having $k$ steps links pore $i$ to pore $j$ ). Fig 12 shows the mean number of drops $n_{\text {drop }}$ between pores and their translated counterparts. For a low polydispersity degree, the mean number of pressure drops $n_{\text {drop }}$ is between the mean number obtained with a Kelvin structure $\left(n_{\mathrm{drop}} / N_{p}^{1 / 3}=1 / 2^{1 / 3} \approx 0.79\right)$ and that obtained with a Weaire Phelan structure $\left(n_{\mathrm{drop}} / N_{p}^{1 / 3}=1\right)$. When the polydispersity degree increases, the number of steps to join two points separated by a period decreases. Rigorously, this parameter applies for open-cell structures but the cluster of pores that forms during the percolation process can benefit from the existence of shortcuts (to progress more quickly). Now, we turn our interest into the estimation of the percolation threshold. For ordered networks (3D lattices) for which $N_{v}$ is constant, Galam and Mauger [39] found an approximate formula linking $x_{p}$ to $N_{v}: x_{p, G M}=0.754\left[\frac{2}{3}\left(N_{v}-1\right)\right]^{-0.9346}$. This expression predicts that the percolation threshold decreases as $N_{v}$ increases, but our results [Figs. 6 and 11(a)] show a contrary trend between the number of neighboring pores $\left\langle N_{v}\right\rangle$ and $x_{p}$. In tree-like networks where loops can be neglected [25, 40, 41] (i.e. there is an unique path linking any two nodes or pores), the percolation threshold is given by: 


$$
x_{p, t}=\frac{\left\langle N_{v}\right\rangle}{\left\langle N_{v}^{2}\right\rangle-\left\langle N_{v}\right\rangle}=\frac{1}{\left\langle N_{v}\right\rangle\left(1+C_{N_{v}}^{2}\right)-1} .
$$

When applied to ordered networks (such as the structure of an ordered monodisperse foam), Eq. (12) yields $x_{p, t}=\left(N_{v}-1\right)^{-1}$ and leads to underestimate the percolation threshold $\left(x_{p, G M} / x_{p, t}=1.101\left[N_{v}-1\right]^{0.0654}>1\right)$. Note that for ordered networks, the percolation threshold can be written as: $x_{p, G M}=0.754\left[\frac{3}{2} x_{p, t}\right]^{0.9346}$. Extend this relationship to the case of polydisperse structures leads to:

$$
x_{p} \approx 0.754\left[\frac{3}{2} \frac{\left\langle N_{v}\right\rangle}{\left\langle N_{v}^{2}\right\rangle-\left\langle N_{v}\right\rangle}\right]^{0.9346} .
$$

Inset of Fig. 11.a) shows that this approximation underestimates the percolation thresholds when the polydispersity degree increases. A linear relationship between $x_{p}$ and the structural parameter $\left\langle N_{v}\right\rangle /\left(\left\langle N_{v}^{2}\right\rangle-\left\langle N_{v}\right\rangle\right)$ could be more appropriate. However, it is worth mentioning that our simulations are performed on structures that are too small to determine an accurate value of the percolation threshold 42 .

Other expressions for the percolation threshold have been suggested in literature [41, 43. For example, the percolation threshold can be approximated by the largest eigenvalue of the adjacency matrix or a matrix combining the adjacency matrix and the degree matrix (which is a diagonal matrix whose elements are equal to the degree of the nodes, i.e. the number of neighboring pores). In some circumstances [41, these estimators can be more accurate than the naive estimator $x_{p, t}$. Alternatively, in fractured porous media, percolation threshold expressions can be derived from the concept of "excluded volume" 44. In such porous media, it is shown that the number of fractures per excluded volume controls the percolation [45.

In the case " $r_{o}$ cst", the permeability $K$ exhibits a linear dependence on the fraction of open windows $x_{\mathrm{ow}}$ (except for $x_{\text {ow }}$ close to the percolation threshold $x_{p}$ ). This result is in good agreement with the behavior of ordered lattice networks. Indeed, in ordered lattice networks, if the fraction of open windows $x_{\mathrm{ow}}$ is not too close the percolation threshold $\left(x_{\mathrm{ow}}>3 / N_{v}\right)$, the permeability $K\left(x_{\mathrm{ow}}\right)$ is well predicted by the effective medium approximation (EMA) [16]: $K\left(x_{\text {ow }}\right) / K(1)=$ $\left(x_{\text {ow }}-x^{\prime}\right) /\left(1-x^{\prime}\right)$ with $x^{\prime}=2 / N_{v}$. However, this relationship predicts an effect of the pore-network structure through the term $x^{\prime}$. But, our pore-network simulations show that the relationship between $K$ and $x_{\text {ow }}$ is almost independent on the polydispersity degree $C_{d}$. This result could be interpreted by invoking two phenomena having opposite effects on permeability. (1) The first one involves the effect of disorder on the percolation threshold. As the percolation threshold decreases when the polydispersity degree increases, it can be expected, at fraction of open windows constant, that the permeability is all the higher as the polydispersity degree is high. (2) However, it is observed in ordered structure that a decrease of the connectivity degree reduces permeability. Therefore, the decrease of $\left\langle N_{v}\right\rangle$ when the polydispersity degree increases should also contribute to reduce the permeability.

Concerning the case " $t_{o}$ cst", the permeability depends not only on $x_{\text {ow }}$ but also on the polydisperse $C_{d}$ as shown on Fig,11. (c). In ordered foams, the dispersion of local conductances induces a reduction of ratios $K\left(x_{\mathrm{ow}}\right) / K(1)$, and the effect is more pronounced when the dispersion is important 10. This effect is shown on Fig. 11.c) by comparing the both curves " $t_{o}$ cst" and " $r_{o}$ cst" at low $C_{d}$. This effect, observed for low polydispersity, is enhanced when the polydispersity degree increases because the dispersion of local conductances increases in the same time.

\section{DISCUSSIONS}

In this section, we discuss various points regarding the permeability of fully open-cell polydisperse foams. First, the use of the mean pressure field assumption to calculate the foam permeability is discussed. Then, we specifically address two main questions associated with the case " $t_{o}$ cst": how do small pores contribute to permeability? Is it possible to define a Kelvin foam equivalent size from the pore-size distribution, i.e. to determine the pore size of a foam having a Kelvin structure and exhibiting the same permeability as the original polydisperse foam?

\section{A. Mean pressure field approximation}

As shown in Sec. II A, the permeability can be calculated from expressions depending on the pressure difference $\Delta P_{i}$ associated with each window [see Eqs. A2 and $[\mathrm{A} 6 \mathrm{~B}]$. Consequently, the accuracy of the permeability prediction depends on the accuracy of the pore pressure prediction. The most simple way to estimate the pore pressure is to consider the mean pressure field approximation for which the pressure of a pore located to the position $z$ is equal to $\lambda z+$ cst. We introduce the random fluctuations $P_{i}^{*}$ of pressure around the mean pressure field and write $P_{i}=\lambda z+P_{i}^{*}+$ cst. Therefore, the pressure drop $\Delta P_{i}=P_{i+}-P_{i-}$ associated with the window " $i$ " is equal to: $\Delta P_{i}=\lambda \Delta z_{i}+\Delta P_{i}^{*}$ where $\Delta z_{i}=z_{i+}-z_{i-}>0$ [see Fig. 2[c)]. Figure 13(a) shows the evolution of the distribution of $\Delta P_{i}^{*} /\left(\lambda d_{3,0}\right)$ for various polydispersity degree $C_{d}$. It is seen that the dispersion increases with $C_{d}$ and that values fluctuate around 0 . Note that the average $\left\langle\Delta P^{*}\right\rangle_{w}$ is different from 0 as the polydispersity degree increases [Fig. 13(b)]. In addition, the squared fluctuating term $\left\langle\left|\Delta P^{*}\right|^{2}\right\rangle_{w}$ [involved in the corrective term $\mathcal{E}_{\text {error }}$, see Eq. (20)], increases as the polydispersity degree increases [Fig. 13 (c)].

Using $\Delta P_{i}=\lambda \Delta z_{i}+\Delta P_{i}^{*}$, we can rewrite Eqs. 

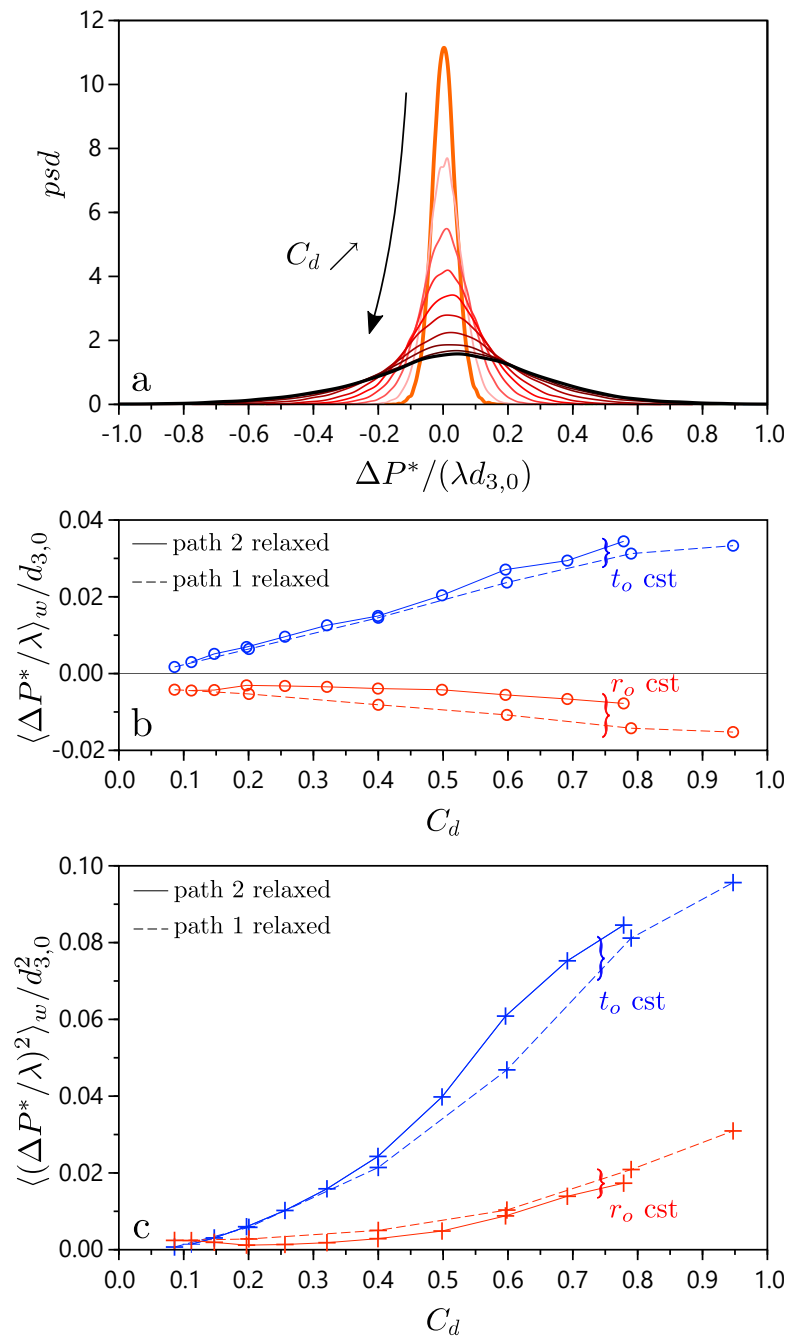

Figure 13. Normalized pressure drop fluctuations $\Delta P^{*} /\left(\lambda d_{3,0}\right)$ : (a) distribution, (b) mean value $\left\langle\Delta P^{*}\right\rangle_{w}$ against $C_{d},(\mathrm{c})\left\langle\left|\Delta P^{*}\right|^{2}\right\rangle_{w}$ against $C_{d}$.

and $(6)$ as:

$$
\begin{aligned}
\frac{K}{\rho_{w} \mu} & =\left\langle G|\Delta z|^{2}\right\rangle_{w}+\left\langle G \frac{\Delta P^{*} \Delta z}{\lambda}\right\rangle_{w} \\
\frac{K}{\rho_{w} \mu} & =\left\langle G|\Delta z|^{2}\right\rangle_{w}+2\left\langle G\left[\frac{\Delta P^{*} \Delta z}{\lambda}+\frac{\left|\Delta P^{*}\right|^{2}}{\lambda^{2}}\right]\right\rangle_{w}
\end{aligned}
$$

The first term $\left\langle G(\Delta z)^{2}\right\rangle_{w}$ in the right-hand side appears in both equations and leads to the following expression of the permeability $K_{\mathrm{mf}}$ with the mean pressure field approximation:

$$
K_{\mathrm{mf}}=\frac{\left\langle N_{v}\right\rangle}{\pi d_{3,0}^{3}}\left\langle r_{o}^{3}(\Delta z)^{2}\right\rangle_{w} .
$$

Note that $\Delta z_{i}$ is related to the inter-pore distance $\delta_{p, i}$ and the angle $\theta_{i}$ between the inter-pore direction and the direction of the macroscopic pressure gradient [Fig. 2(c)]: $\Delta z_{i}=\delta_{p, i} \cos \left(\theta_{i}\right)$. For random isotropic structure, $\delta_{p, i}$ and $\theta_{i}$ are independent random variables and
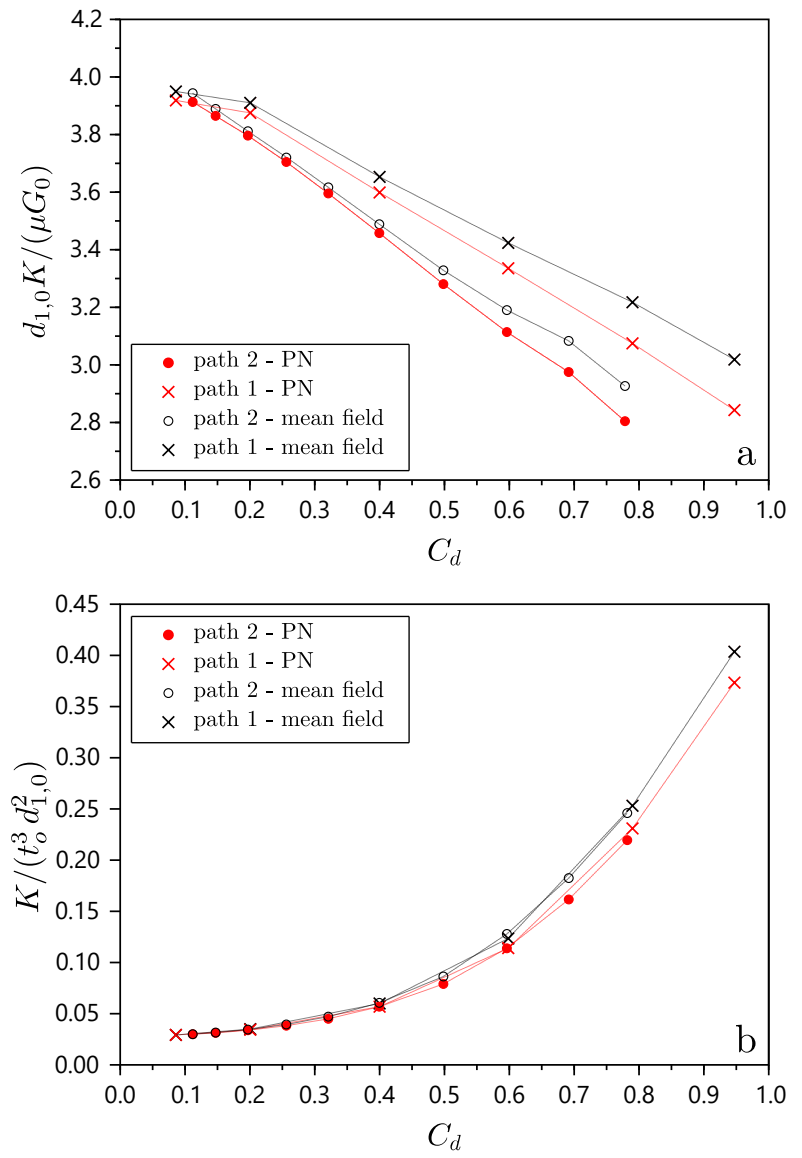

Figure 14. Comparison between pore-network (PN) simulations on relaxed structures and permeability calculations from mean field hypothesis [Eq. [16]]: (a) constant aperture size " $r_{o}$ cst", (b) constant aperture ratio " $t_{o}$ cst".

$\left\langle\cos ^{2}(\theta)\right\rangle_{w}=\frac{1}{3}$. Therefore, we have that $\left\langle r_{o}^{3}(\Delta z)^{2}\right\rangle_{w}=$ $\frac{1}{3}\left\langle r_{o}^{3} \delta_{p}^{2}\right\rangle_{w}$. Moreover, Eq. [16 applies to highly porous foams ("dry foam limit"), i.e. $\phi=1$. In the case where the porosity $\phi$ is less than 1 , the values of permeability given by Eq. (16) must be multiplied by $\phi$.

Figure 14 shows that the permeabilities estimated with the mean field approximation [Eq. $\sqrt{16}$ are quite accurate for the cases " $r_{o}$ cst" and " $t_{o}$ cst". The relative deviation $\left(K_{\mathrm{mf}} / K_{\mathrm{PN}}\right)-1$ between the mean field prediction $K_{\mathrm{mf}}$ and the pore-network calculation $K_{\mathrm{PN}}$ is less than $12 \%$ on the variation range of $C_{d}$ used in the simulations on relaxed structures. In the case " $t_{o}$ random", Fig. 15 shows that the values of permeability calculated with the mean field assumption evolve as $\left\langle t_{o}^{3}\right\rangle_{A}=\left\langle t_{o}\right\rangle^{3}\left(1+\left(\Delta t_{o} /\left\langle t_{o}\right\rangle\right)^{2}\right)$, contrary to porenetwork results (Fig. 10), and become less accurate as the variation $\Delta t_{o}$ of the aperture ratio or the polydispersity degree $C_{d}$ increases.

Specific expressions for $K_{\mathrm{mf}}$ can be calculated for each 

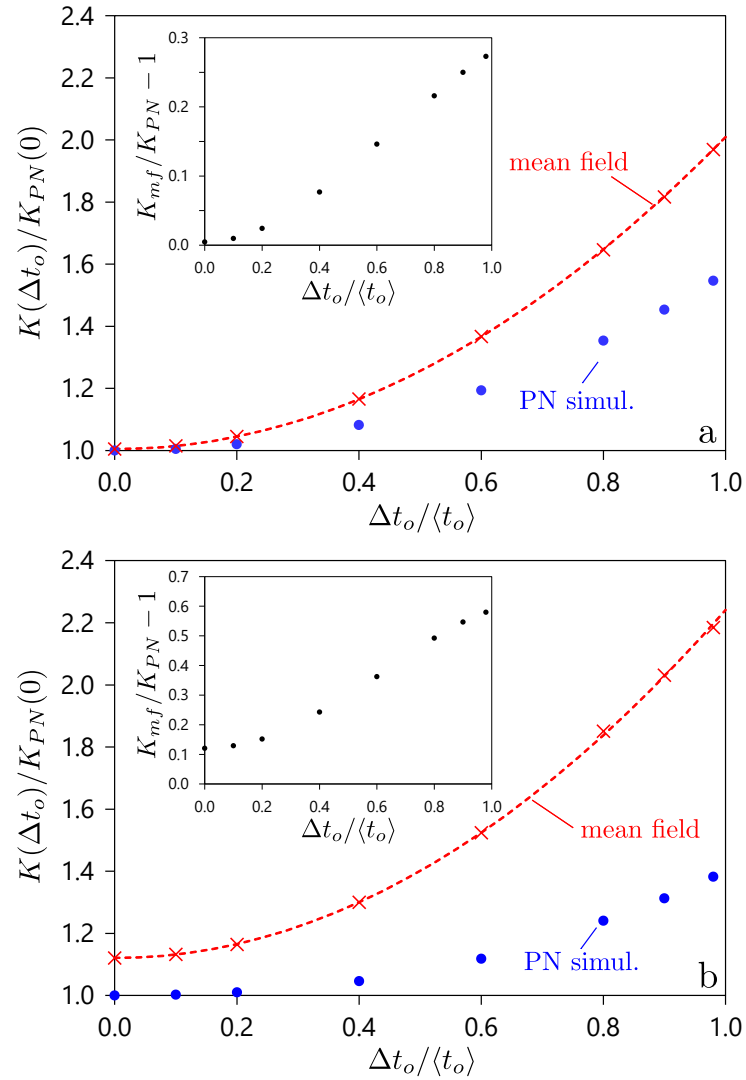

Figure 15. Comparison between pore-network simulations and permeability calculations from mean field hypothesis in the case " $t_{o}$ random" and relaxed "path 2": (a) $C_{d}=0.11$, (b) $C_{d}=0.78$. Inset graphs correspond to the relative prediction error with the mean field hypothesis. Dashed lines are calculated with $K_{\mathrm{mf}}\left(\Delta t_{o}\right)=K_{\mathrm{mf}}(0)\left[1+\left(\Delta t_{o} /\left\langle t_{o}\right\rangle\right)^{2}\right]$ while crosses correspond to the calculations performed with the data associated with the randomly drawn configurations used for the pore network simulations.

case considered in the simulations:

$$
\begin{aligned}
& K_{\mathrm{mf}}=\frac{3}{\pi}\left\langle N_{v}\right\rangle \frac{\mu G_{0}}{d_{3,0}^{3}}\left\langle|\Delta z|^{2}\right\rangle_{w}, \text { for " } r_{o} \text { cst", } \\
& K_{\mathrm{mf}}=\frac{\left\langle N_{v}\right\rangle}{\pi d_{3,0}^{3}}\left\langle r_{w}^{3}|\Delta z|^{2}\right\rangle_{w} t_{o}^{3}, \text { for " } t_{o} \text { cst", } \\
& K_{\mathrm{mf}}=\frac{\left\langle N_{v}\right\rangle}{\pi d_{3,0}^{3}}\left\langle r_{w}^{3}|\Delta z|^{2}\right\rangle_{w}\left\langle t_{o}^{3}\right\rangle_{w}, \text { for " } t_{o} \text { random". }
\end{aligned}
$$

The permeability expressions [Eqs. (17), (18) and [19] ] deduced from the mean field hypothesis give simple analytical expressions revealing the influence of morphological parameters. For example, in the case of a constant aperture ratio " $t_{o}$ cst", it appears that the permeability depends on $\left\langle r_{w}^{3}|\Delta z|^{2}\right\rangle_{w}=\left\langle r_{w}^{3}\right\rangle_{w}\left\langle|\Delta z|^{2}\right\rangle_{w}+$ $\operatorname{cov}\left(r_{w}^{3},|\Delta z|^{2}\right)$ where $\operatorname{cov}(.,$.$) is the covariance of ran-$ dom variables associated with windows. The random variables $r_{w}^{3}$ and $\Delta z$ are not independent and their covariance is strictly positive, as shown in Fig. 16. Therefore, the increase of permeability with $C_{d}$ shown in Fig. 8(b)

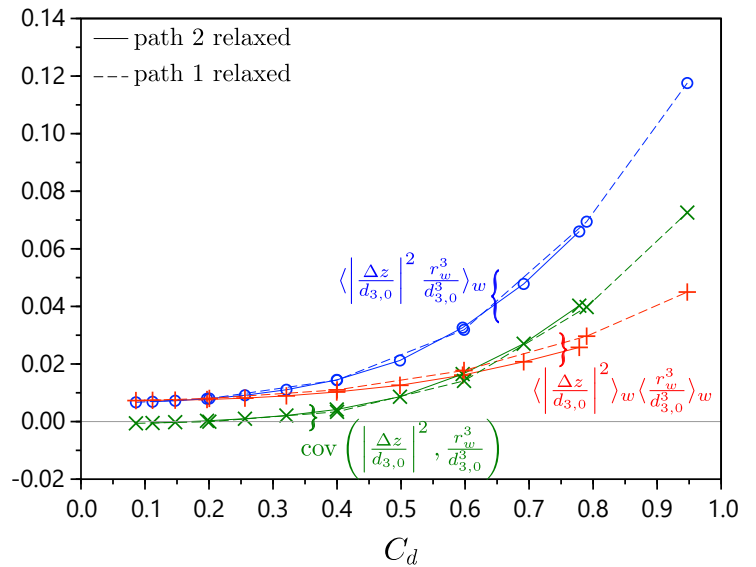

Figure 16. $\left\langle r_{w}^{3}|\Delta z|^{2}\right\rangle_{w},\left\langle r_{w}^{3}\right\rangle_{w}\left\langle|\Delta z|^{2}\right\rangle_{w}$ and $\operatorname{cov}\left(r_{w}^{3},|\Delta z|^{2}\right)$ normalized by $d_{3,0}^{5}$ as a function of $C_{d}$ in the case " $t_{o}$ cst".

is the cumulative effect of the evolution of the product $\left\langle r_{w}^{3}\right\rangle_{w}\left\langle|\Delta z|^{2}\right\rangle_{w}$ and the covariance $\operatorname{cov}\left(r_{w}^{3}|\Delta z|^{2}\right)$ for the main part when $C_{d}>0.65$.

We now consider the modeling of the corrective term $\mathcal{E}_{\text {error }}$ of the mean field approximation. In Eqs. (14) and (15), the second terms in the right-hand sides are two different expressions for the corrective term to be applied to the mean pressure field approximation. Requiring these expressions to be equal implies that the corrective term must be negative:

$$
\frac{1}{\lambda}\left\langle G \Delta P^{*} \Delta z\right\rangle_{w}=-\frac{1}{\lambda^{2}}\left\langle G\left(\Delta P^{*}\right)^{2}\right\rangle_{w}<0 .
$$

Consequently, the permeability calculated with the mean pressure field approximation is an upper bound for the permeability (as confirmed by our pore-network simulations). This result was shown previously in Ref. Durand et al. 24.

Finally, the error in the permeability calculation with the mean pressure field approximation can be written as follows:

$$
K-K_{\mathrm{mf}}=-\frac{\left\langle N_{v}\right\rangle}{\pi} d_{3,0}^{2} \mathcal{E}_{\text {error }},
$$

with $\mathcal{E}_{\text {error }}=\left\langle r_{o}^{3}\left(\Delta P^{*} / \lambda\right)^{2}\right\rangle_{w} / d_{3,0}^{5}$.

In the cases " $t_{o}$ cst" and " $t_{o}$ variable", the aperture sizes $r_{o}$ and the fluctuating term $\Delta P^{*}$ are not independent random variables, and consequently, $\left\langle r_{0}^{3}\left|\Delta P^{*}\right|^{2}\right\rangle_{w}=$ $\left\langle r_{o}^{3}\right\rangle_{w}\left\langle\left|\Delta P^{*}\right|^{2}\right\rangle_{w}+\operatorname{cov}\left(r_{o}^{3},\left|\Delta P^{*}\right|^{2}\right)$. Figure 17 shows the different contributions to the error term $\mathcal{E}_{\text {error }}$ for the case " $t_{o}$ cst". The main contribution to the error comes from the product $\left\langle r_{o}^{3}\right\rangle_{w}\left\langle\left|\Delta P^{*}\right|^{2}\right\rangle_{w}$, and the contribution from the covariance increases as the polydispersity degree increases. The mean field hypothesis assuming a decorrelation between the pressure field and the porenetwork structure is no longer possible when the degree of polydispersity is high and/or the fluctuations of aperture ratios are important. In this case, finding a strong correction to the mean field predictions involving disorder parameters (such as $C_{d}, C_{N_{v}}$ or $\left[d_{3,0} / d_{2,0}\right]-1$ ) and 


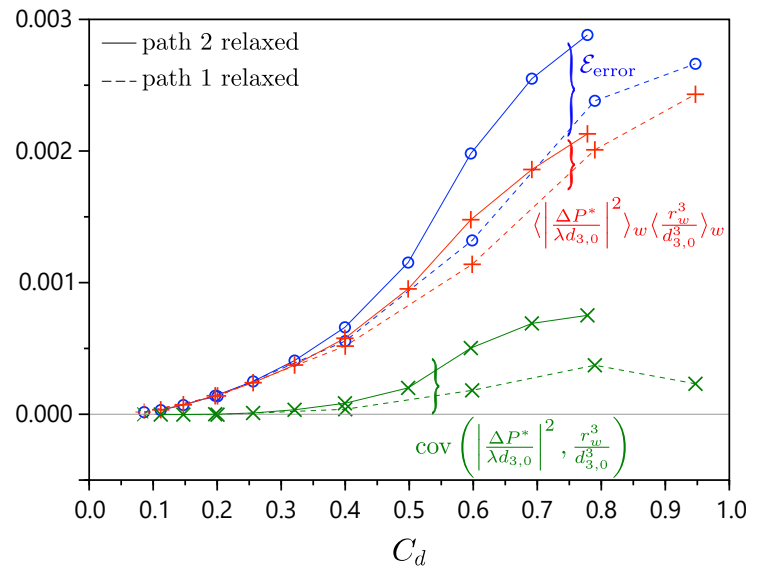

Figure 17. Error sources in permeability predictions with mean field approximation against $C_{d}$ for the case " $t_{o}$ cst". Note that Figs. 16 and 17 give complementary information leading to the relative error $\left(K-K_{\mathrm{mf}}\right) / K_{\mathrm{mf}}=$ $-\left\langle r_{w}^{3}\left|\Delta P^{*} / \lambda\right|^{2}\right\rangle_{w} /\left\langle r_{w}^{3}|\Delta z|^{2}\right\rangle_{w}$

dimensionless parameter $\left(\operatorname{such} N_{v}\right.$ ) is of interest. To this end, and with the aim of estimating the disorder induced by the variation of the local conductance over the rough surface, we introduce a specific disorder parameter defined as: $C_{G}=\sigma_{G} /\langle G\rangle_{w}$, where $\sigma_{G}$ is the standard deviation of the local conductance. Assuming the validity of the Sampson's law, $C_{G}$ is equal to the normalized standard deviation of the window aperture size raised to the power of 3: $C_{G}=\sigma_{r_{o}^{3}} /\left\langle r_{o}^{3}\right\rangle_{w}$. Hereafter, we use $\mathrm{A}=\left[d_{3,0} / d_{2,0}\right]-1, B=C_{G}$ and $C=C_{N_{v}}$, to build an approximate formula for $d_{3,0}^{3} \mathcal{E}_{\text {error }} /\left\langle r_{o}^{3}\right\rangle_{w}$ that we will denote by $\mathcal{R}_{\text {error }}$ :

$$
\begin{aligned}
\mathcal{R}_{\text {error }}= & \left\langle\frac{r_{o}^{3}}{\left\langle r_{o}^{3}\right\rangle_{w}}\left(\frac{\Delta P^{*}}{\lambda d_{3,0}}\right)^{2}\right\rangle_{w} \\
\approx & 0.118 \mathrm{BC}+4.101 \mathrm{AC}-0.207 \mathrm{AB} \\
& -0.578 \mathrm{C}^{2}+0.012 \mathrm{~B}^{2}-8.656 \mathrm{~A}^{2} \\
& -0.159 \mathrm{C}+0.034 \mathrm{~B}-0.159 \mathrm{~A} .
\end{aligned}
$$

Figure 18 compares the values of $R_{\text {error }}$ found by porenetwork simulations to the values estimated by using Eq. (22). The coefficient of determination $R^{2}$ is equal to 0.999 .

Finally, an improved version of the permeability formula based on the mean pressure field assumption can be derived from Eqs. (16) and (22):

$$
K=\frac{\left\langle N_{v}\right\rangle}{\pi d_{3,0}^{3}}\left[\left\langle r_{o}^{3}(\Delta z)^{2}\right\rangle_{w}-d_{3,0}^{2}\left\langle r_{o}^{3}\right\rangle_{w} \mathcal{R}_{\text {error }}\right] .
$$

\section{B. Effect of small pores}

In this section, we investigate the effect of small pores on the permeability of open-cell foam samples under the

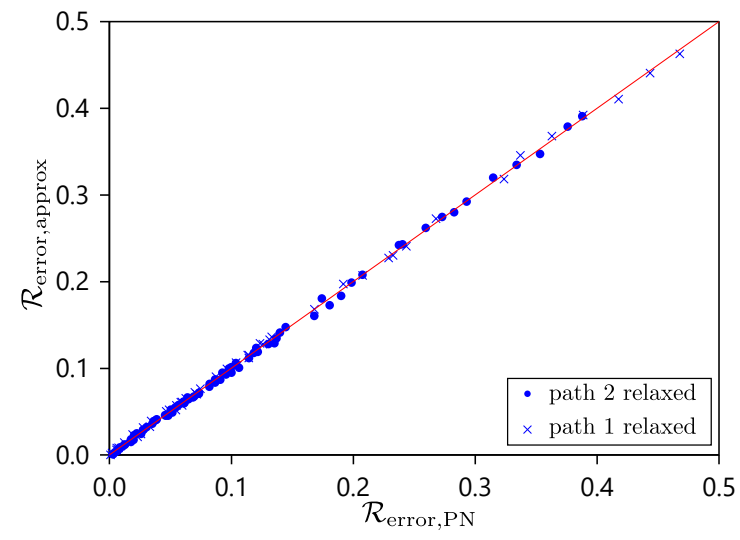

Figure 18. Comparison between $\mathcal{R}_{\text {error }}$ calculated by porenetwork simulations (" $t_{o}$ cst" and " $t_{o}$ random") for all foam relaxed structures ("path 1" and "path 2") to values calculated with the approximation given by Eq. 22.

assumption of constant aperture rate (" $t_{o}$ cst"). In polydisperse structures, small pores are numerous but do not occupy a large volume. This common result can be illustrated by using the cumulative pore-size distribution with various weightings. These cumulative pore-size distributions can be defined as follows:

$$
F_{n}(d)=\frac{\sum_{i \text { where } d_{p, i} \leq d}\left[d_{p, i}^{n}\right]}{\sum_{i=1}^{N p, t}\left[d_{p, i}^{n}\right]},
$$

where $N_{p, t}$ is the total number of pore.

By considering $n=0$, we obtain the proportion in number of pores having a size smaller than $d$ (numberweighted distribution). For $n=3$, we obtain the volume fraction of all the pores smaller than $d$ (volume-weighted distribution). The different cumulative pore-size distributions are almost identical in structures having a low polydispersity degree, as shown in Fig. 19(a). On the contrary, for structures having a high polydispersity degree, the volume-weighted distribution is wider than the number-weighted distribution [Fig. 19(b)]. As previously pointed and illustrated in Fig. 19. b), small pores are numerous but do not occupy a large volume in structures having a high polydispersity degree. For example, $61 \%$ of small pores in number fill only $4 \%$ of the pore space when $C_{d}=0.78$.

To highlight the effect of small pores on the permeability of open cell foam samples, pore network simulations were performed on structures for which pores smaller than a threshold size $d_{\mathrm{cp}}$ are considered to be completely closed (i.e. their windows are closed or all their window conductances are set to be equal to zero). As expected, the permeability values decreases while the threshold size $d_{\text {cp }}$ increases [Fig. 20]. Note that the permeability tends to zero before all the pores are closed. This is brought about by the vanishing of percolation (site percolation) which occurs when the closed pores number is enough to stop the flow of fluid.

Now, we focus more specifically on the case where 
the volume fraction of small closed pores is low (and $\left.d_{\mathrm{cp}}<d_{1,0}\right)$. Figs. 20 and 19 show that a decrease of the permeability value equal to $5 \%$ is obtained by closing: $2 \%$ of pores in volume (or $5 \%$ in number) for structures having a low polydispersity degree $\left(C_{d}=0.11\right)$, and $4 \%$ of pores in volume (or $63 \%$ in number) for structures having a high polydispersity degree $\left(C_{d}=0.78\right)$. Clearly, the volume fraction of small pores is the key parameter to evaluate the effect of small pores on foam permeability. This result is in good agreement with a model of electric conductivity in a suspension of spheroids due to Fricke [46] and used to model the permeability of fractured rock by Ref. [47]. In this model, the effective permeability of a porous media of permeability $K_{m}$ containing randomly oriented spheroidal inclusions of permeability $\kappa K_{m}$ is equal to

$$
K=K_{m}(1-\beta \phi),
$$

where $\phi$ is the volume fraction of inclusions, and $\beta$ is a coefficient depending on the ratio of permeabilities $\kappa$ and the shape of inclusions ( $\beta=1.5$ for spherical inclusion with zero permeability, $\kappa=0)$.

Fig. 21 shows that our results obtained with structures made of discrete pores follow the relationship given by Fricke's equation with a specific value of $\beta$ for each polydispersity degree.

\section{Equivalent Kelvin foam}

In this section, we restrict the analysis to the case where the aperture ratio is constant (" $t_{o}$ cst"). For such a polydisperse foam, we define an equivalent monodisperse/Kelvin pore size $d_{\text {eq }}$, which is defined as the pore size of a foam sample having a Kelvin structure and exhibiting the same permeability and aperture ratio $t_{o}$ as the initial polydisperse foam sample. In the following, we aim to estimate $d_{\text {eq }}$ from the pore-size distribution of the polydisperse foam sample.

The equivalent Kelvin pore size can be calculated from our pore-network simulation results [Fig. 8(b)] and from Eq. 11 .

$$
d_{\mathrm{eq}}=\left[K_{\mathrm{PN}} /\left(c_{K} t_{o}^{3}\right)\right]^{0.5} .
$$

Different sizes calculated from pore-size distribution were tested to estimate the equivalent Kelvin pore sizes: (i) $d_{m, n}\left(d_{1,0}, d_{3,0}\right.$ and $\left.d_{3,2}\right)$, (ii) two sizes defined from the cumulative pore size functions $\left(F_{2}\right.$ and $F_{3}$ ), (iii) a size defined from the mean volume of pores larger than a threshold size defined from the volume-weighted distribution $F_{3}$.

As the ratio $K / d_{1,0}^{2}$ depends on $C_{d}$ (Fig. 8), the number-weighted mean diameter $d_{1,0}$ is not relevant to estimate $d_{\text {eq }}$. Indeed, the use of $d_{1,0}$ as equivalent Kelvin pore size would lead to underestimate the permeability to an order of magnitude for very polydisperse structures. We also checked that the mean diameter $d_{3,0}$ is not suitable (not shown here). Sauter diameter $d_{3,2}$ seems more
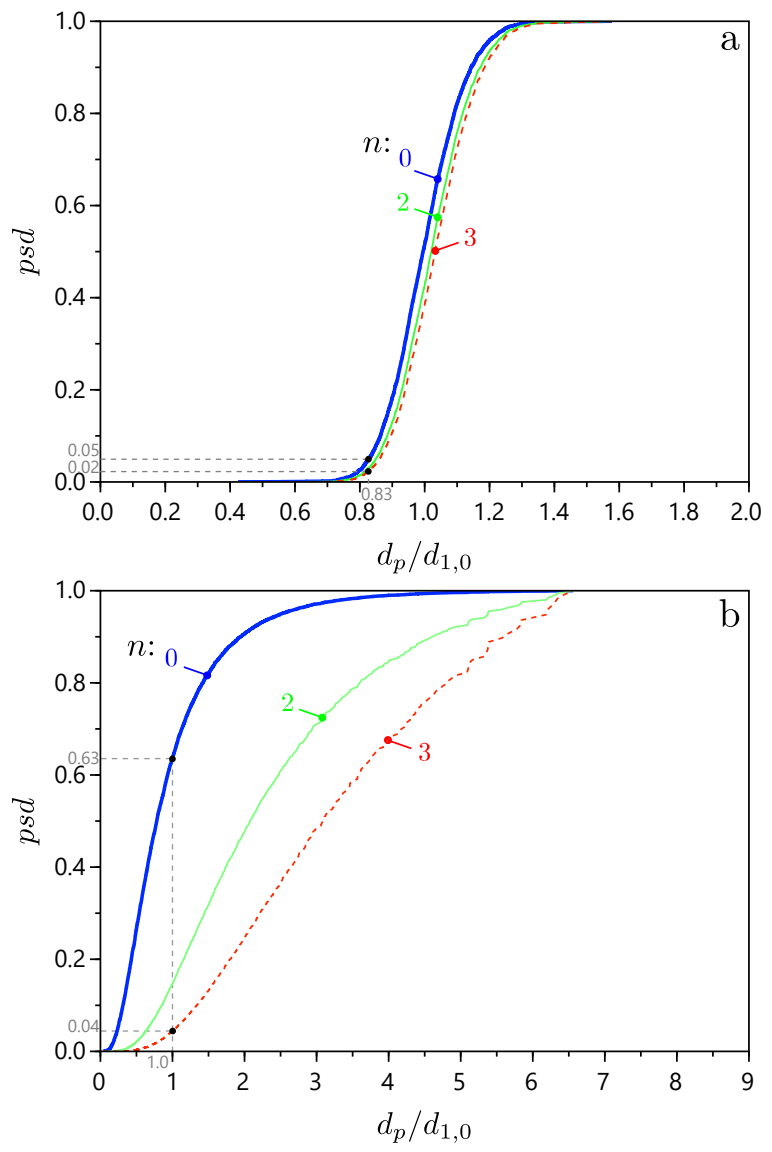

Figure 19. Dependence on normalized pore size $d_{p} / d_{1,0}$ of cumulative pore-size distributions $F_{n}(d)$ with $n=0,2$ or 3 for "path 2" Laguerre structures: (a) $C_{d}=0.11$, (b) $C_{d}=0.78$.

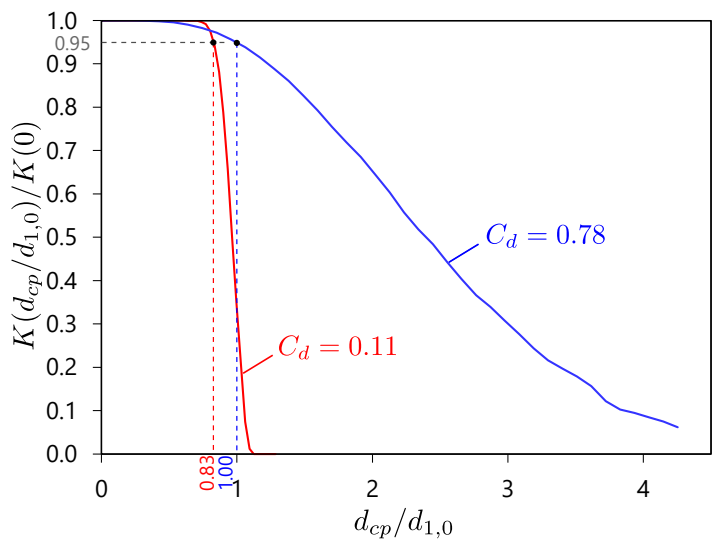

Figure 20. Effect of closing small pores in the case " $t_{o}$ cst" ("path 2" relaxed structures and $C_{d}=0.11$ or 0.78 ). The windows of pores having a pore size less than $d_{\mathrm{cp}}$ are closed.

appropriate as it would lead to overestimate the permeability of about $30 \%$, (see Fig. 22 .

For granular material, Hazen uses the grain size for which the mass of grains smaller than $D_{10}$ is equal to $10 \%$ of the mass of all grains: $F_{3}\left(D_{10}\right)=10 \%$ (by assuming that all grains have the same density). Similarly, 


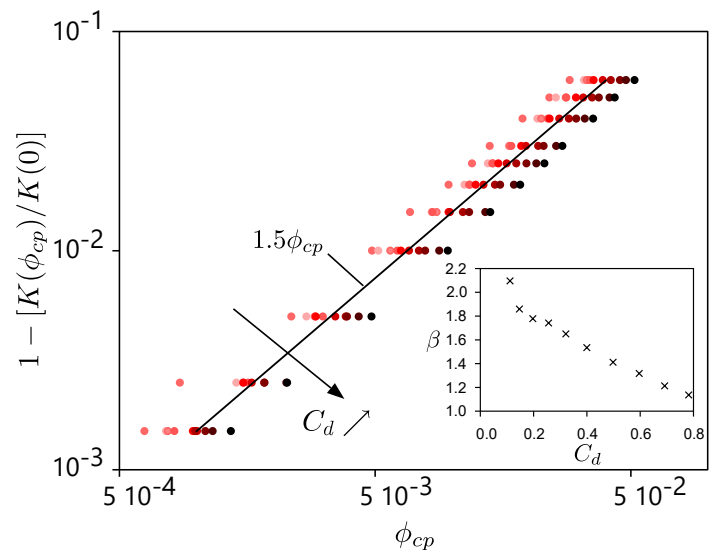

Figure 21. Effect of closing small pores in the case " $t_{o}$ cst" and in the dilute regime $\phi_{c p}=F_{3}\left(d_{\mathrm{cp}}\right) \ll 1$. Points correspond to data obtained with "path 2" relaxed structures and various polydispersity degree $C_{d}$ from 0.11 to 0.78 (each color corresponds to a value of $C_{d}$ ). Full line is plotted by assuming spherical inclusion with zero permeability and $\phi=\phi_{c p}$ in Eq. (25). Inset graph corresponds to values of $\beta$ adjusted for that the predictions of Eq. 25 fit to our data.

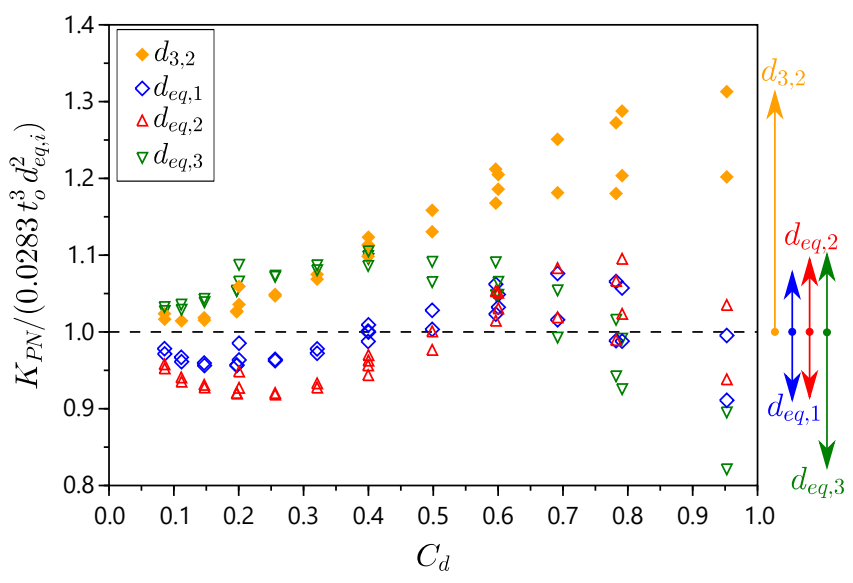

Figure 22. Ratio of the values $K_{\mathrm{PN}}$ of permeability found by pore-network simulations to values of permeability of Kelvin foam having pore sizes equal to $d_{3,2}, d_{\mathrm{eq}, 1}, d_{\mathrm{eq}, 2}$ or $d_{\mathrm{eq}, 3}$ for various $C_{d}$ and all foam structures ("path 1", "path 2", "path 1 relaxed", "path 2 relaxed").

for each cumulative function $\left(F_{2}\right.$ and $\left.F_{3}\right)$, we checked if it is possible to find a unique value $\epsilon$ for which $F_{n}\left(d_{\epsilon}\right)=\epsilon$ and $d_{\epsilon}=d_{\mathrm{eq}}$ for all foam samples with $n=2$ or 3 . From Eq. (26) and the cumulative pore size functions, the values of $\epsilon$ can be calculated for each case, $n=2$ or 3 . Figure 23 shows that no unique value of $\epsilon$ can be found for $F_{2}$ and $F_{3}$. We note however that the values of $F_{2}\left(d_{\mathrm{eq}}\right)$ and $F_{3}\left(d_{\mathrm{eq}}\right)$ remain in a limited range of variations. Therefore, we chose two values in order to define two pore sizes from the cumulative functions, $F_{2}$ and $F_{3}$, giving an estimate of the equivalent Kelvin pore size: $d_{\mathrm{eq}, 2}$ is the size for which $F_{2}\left(d_{\mathrm{eq}, 2}\right)=0.65$ and $d_{\mathrm{eq}, 3}$ is the size for which $F_{3}\left(d_{\mathrm{eq}, 3}\right)=0.43$.

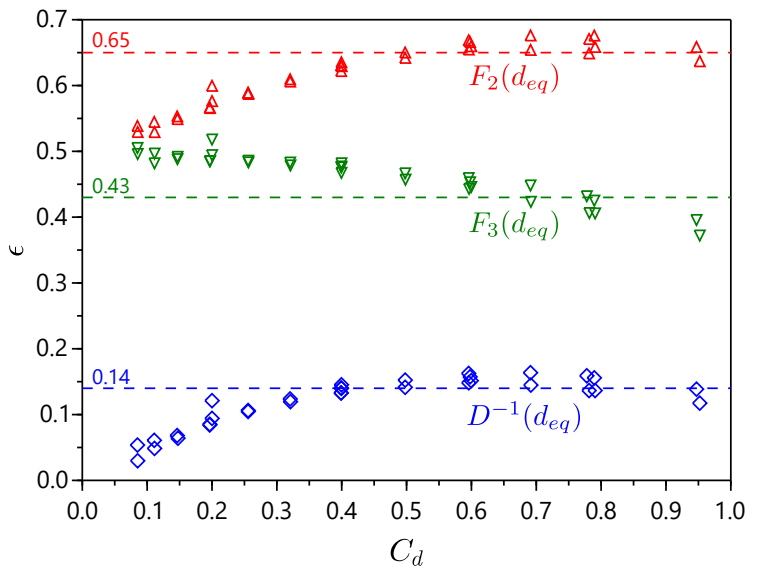

Figure 23. $\epsilon$ against $C_{d}$. Note that the definition of $\epsilon$ depends on the way of calculating the corresponding size $d_{e q, i}: \epsilon=$ $D^{-1}\left(d_{\mathrm{eq}}\right)$ for $d_{\mathrm{eq}, 1}, F_{2}\left(d_{\mathrm{eq}}\right)$ for $d_{\mathrm{eq}, 2}, F_{3}\left(d_{\mathrm{eq}}\right)$ for $d_{\mathrm{eq}, 3}$.

Finally, we define a mean pore size function $D(\epsilon)$, as the size of an average pore of volume equal to the mean volume of the pores having $d_{i}>d_{\epsilon}$ :

$$
D(\epsilon)=\left(\frac{\sum_{i, \text { where } d_{i}>d_{\epsilon}} d_{i}^{3}}{\sum_{i, \text { where } d_{i}>d_{\epsilon}} 1}\right)^{1 / 3} .
$$

where $d_{\epsilon}$ is the size for which $F_{3}\left(d_{\epsilon}\right)=\epsilon$. i.e. $d_{\epsilon}=$ $F_{3}^{-1}(\epsilon)$. The introduction of this pore size function is motivated by the fact that the contribution of small pores to permeability is low.

The function $D(\epsilon)$ depends on the cumulative functions, $F_{1}$ and $F_{3}$, as follows:

$$
D(\epsilon)=d_{3,0}\left(\frac{1-\epsilon}{1-F_{0}\left(F_{3}^{-1}(\epsilon)\right)}\right)^{1 / 3} .
$$

From Eqs. 26) and (28), the values of $\epsilon=D^{-1}\left(d_{\text {eq }}\right)$ can be calculated ( $D^{-1}$ is the inverse function of $\left.D\right)$. As for the others attempts, Fig. 23 shows that no unique value of $\epsilon$ can be found for $D^{-1}\left(d_{\mathrm{eq}}\right)$. However, a value of $\epsilon \approx 0.14$ is in agreement with most of the simulated data for moderately high to high polydispersity degree and can be used to define another approximate of the equivalent pore size $: d_{\mathrm{eq}, 1}=D(0.14)$.

Figure 22 compares the simulated data to the permeability values calculated by using Eq. (11) with $d_{1,0}$ set to be equal to $d_{e q, i}$. From which it becomes apparent that the most accurate definitions to estimate $d_{\text {eq }}$ are $d_{\mathrm{eq}, 1}=D(0.14)$ and $d_{\mathrm{eq}, 2}=F_{2}^{-1}(0.65)$.

Importantly, we note that the equivalent Kelvin pore size does not correspond to a specific pore belonging to the set of all pores and which could be taken as representative of all pores. For example, for a "path 2" polydisperse sample with $C_{d}=0.79$, the pores having a size approximately equal to the equivalent Kelvin pore size have on average about 30 neighboring pores. This is much more than the 14 neighbors expected for a Kelvin structure. 


\section{CONCLUSION}

To study the effects of polydispersity on solid foam permeability, we performed different numerical simulations to generate foam structures (Laguerre tessellations via Neper software, followed by relaxation calculations to minimize the surface area and satisfy Plateau's laws via Surface Evolver software), and to calculate the fluid flow through foam with pore-network simulations by considering either open-cell foam (no closed window) or foam with some closed windows (randomly chosen). Concerning foam microstructures, it appears that different structures sharing the same pore size dispersion (as a Laguerre structure and its relaxed version) can have different morphologies (in a topological sense).

Despite the variety of microstructures generated, certain general conclusions can be drawn concerning the permeability of polydisperse open-cell foam: (1) in the case of constant aperture size (" $r_{o}$ constant"), we show that the permeability can be calculated from parameters derived from a dimensional analysis and a dimensionless function $\mathcal{F}$ depending on $\left\langle N_{v}\right\rangle /\left(\left\langle N_{v}^{2}\right\rangle-\left\langle N_{v}\right\rangle\right)$ (see Fig. 9); (2) in the case of constant aperture ratio " $t_{o}$ constant" (i.e. the apertures of windows connecting neighboring pores increase as the associated pore sizes), permeability at constant mean pore size increases as the polydispersity degree increases and in a similar way for all foam microstructures (as Laguerre structures and their relaxed versions) ; (3) as long as the dispersion of the window aperture rate remains low, the assumption of mean pressure field is accurate enough to calculate the permeability [Eqs. (18) and (19)] from key microstructural parameters, $d_{3,0}, t_{o}, r_{w},\left\langle N_{v}\right\rangle$ and $\Delta z$ (however, their characterization requires $3 \mathrm{~d}$ tomography) ; (4) in highly polydisperse structures, small pores do not contribute significantly to the global permeability (due to their low volume fraction).

Concerning polydisperse foams containing closed windows, the percolation threshold $x_{p}$ decreases with the polydispersity degree while the mean number of neighbors decreases; and the parameter $\left\langle N_{v}\right\rangle /\left(\left\langle N_{v}^{2}\right\rangle-\left\langle N_{v}\right\rangle\right)$ was helpful for establishing an explicit link to the microstructure. Moreover, in the case of constant aperture size (" $r_{o}$ constant"), the relationship between the permeability and the fraction of open windows is very insensitive to the polydispersity degree itself [Fig. 11(b)]. We interpret this observation as the result of two opposite mechanisms resulting from polydispersity: the decrease of percolation threshold $x_{p}$ accompanied by a lower mean number of neighbors $\left\langle N_{v}\right\rangle$. Further study on percolation of polydisperse foams will be necessary to build a complete analytical model as done for monodisperse and ordered foams. For this work, the extension of the Kirkpatrick's model due to Klimenko et al. [48, will be considered.

Finally, while the following results may be general in character for polydisperse open-cell foams, they will have to be confirmed on other realistic microstructures:
(1) the correction of the permeability calculations based on the mean pressure field assumption [Eq. [22]], (2) the empirical estimate of the equivalent Kelvin size from pore-size distributions $\left(d_{\mathrm{eq}, 1}\right.$ and $\left.d_{\mathrm{eq}, 2}\right)$ when a constant aperture ratio " $t_{o}$ constant" is assumed.

\section{Appendix A: Alternative permeability calculations}

In this appendix, we present the derivation of two alternative expressions for permeability. One calculation is based on the definition of permeability, and the second approach involves energetic considerations.

In Eq. (4), the permeability is derived from the global flow rate $Q_{z}$ calculated from a sum of local flow rates passing through any cross-section, $A(z)$. Consequently, we can write:

$$
\begin{aligned}
Q_{z} & =\frac{1}{H} \int_{0}^{H} Q_{z} d z=\frac{1}{H} \int_{0}^{H}\left[\sum_{i \in A(z)} q_{i}\right] d z \\
& =\frac{1}{H} \sum_{i \in\{w\}} q_{i} \Delta z_{i},
\end{aligned}
$$

where $\Delta z_{i}=z_{i+}-z_{i-}>0$ [see Fig. 2(c)], and $\sum_{i \in A(z)}$ and $\sum_{i \in\{w\}}$ are respectively the sum over all windows covering the rough surface and the sum over all the windows contained in the whole structure. Note that a window $i$ is active in the integral calculation as long as the position of the cross-section is between $z_{i-}$ and $z_{i+}$.

From this calculation, we derive the first alternative expression for permeability:

$$
K_{\mathrm{av}}=\frac{\mu}{V \lambda} \sum_{i \in\{w\}} G_{i} \Delta P_{i} \Delta z_{i},
$$

where $V=A H$ is the volume of the elementary structure.

Another expression for permeability can be derived from energetic considerations. This expression is easily understood by considering the analogy between fluid flow through the pore-network and the electrical current transport through a network of wires. Here, $\Delta P_{i}$ can be seen as the equivalent of the tension $U_{i}, q_{i}$ as the analogous of the current $I_{i}, G_{i}$ can be viewed as the electrical conductance $G_{e, i}$, and $K / \mu$ is the equivalent of the electrical conductivity $\sigma_{e}$. In a network of electrical resistances, the dissipated energy by unit of time $\mathcal{P}$ can be expressed as a function of the equivalent conductance of the network $G_{\mathrm{e}, \mathrm{eff}}$ and the power dissipated locally [24]:

$$
\mathcal{P}=G_{e, \mathrm{eff}} U_{\mathrm{m}}^{2}=\sum_{i \in\{w\}} G_{e, i} U_{i}^{2}
$$

where $U_{m}=\lambda_{e} H$ is the tension applied between the boundaries of the network.

The equivalent conductance of the network $G_{e, \text { eff }}$ is related to the effective conductivity of the network as 
follows:

$$
\sigma_{e}=\frac{H}{A} G_{e, \mathrm{eff}}=\frac{1}{V \lambda_{e}^{2}} \sum_{i \in\{w\}} G_{e, i} U_{i}^{2} .
$$

When expressed in terms of the effective fluid flow conductance $G_{\text {eff }}$ and permeability $K$, Eq. (A4) leads to:

$$
\begin{aligned}
G_{\mathrm{eff}} & =\frac{1}{\Delta P_{\text {macro }}^{2}} \sum_{i \in\{w\}} G_{i}\left|\Delta P_{i}\right|^{2}, \\
K_{e}=\mu \frac{H}{A} G_{\mathrm{eff}} & =\frac{\mu}{V \lambda^{2}} \sum_{i \in\{w\}} G_{i}\left|\Delta P_{i}\right|^{2} .
\end{aligned}
$$

The equivalence between Eqs. (A2) and (A6) requires that:

$$
\sum_{i \in\{w\}} q_{i} \lambda \Delta z_{i}=\sum_{i \in\{w\}} q_{i} \Delta P_{i} .
$$

In the context of electrical current transport, the above equation is equivalent to:

$$
\sum_{i \in\{w\}} I_{i} \lambda_{e} \Delta z_{i}=\sum_{i \in\{w\}} I_{i} U_{i}
$$

Eqs. A7 and (A8) can be justified by invoking the Tellegen's theorem, since both $\lambda_{e} \Delta z_{i}$ and $U_{i}$ satisfy the Kirchhoff's voltage law 49 .

Unlike Eq. (4), Eqs. $\overline{\mathrm{A} 2}$ and $(\mathrm{A} 6$ ) involve sums over the windows contained in the whole volume. By introducing the volume density of windows $\rho_{w}$ in Eqs. (A2) and (A6), Eqs. 144) and (15) are recovered.

\section{Appendix B: Details about microstructures}

In this appendix, we give, for interested readers, some details concerning our numerical foam microstructures (surface free energy density, number of neighboring pores, local conductances, inter-pore distance and covariances between various microstructural parameters).

\section{Surface free energy density}

Kraynik et al. [27] introduced a polydispersity parameter $p$ defined as $p=\left(\left\langle d_{p}^{3}\right\rangle^{2 / 3} /\left\langle d_{p}^{2}\right\rangle\right)-1$. With the assumption of constant sphericity, the parameter $p$ is related to the surface free energy density $E$ (defined as $E=\sigma \sum S_{w} / \sum V_{p}$ with $\sigma$ the tension surface) of the relaxed foam through:

$$
E=\frac{\sigma}{\left\langle V_{p}\right\rangle^{1 / 3}} \frac{(36 \pi)^{1 / 3}}{\langle\Psi\rangle} \frac{1}{1+p},
$$

with $\langle\Psi\rangle=0.91$.

Kraynik and co-authors have shown that this theoretical relationship between the surface free energy density $E$ and $p$ is remarkably well satisfied in a large variety

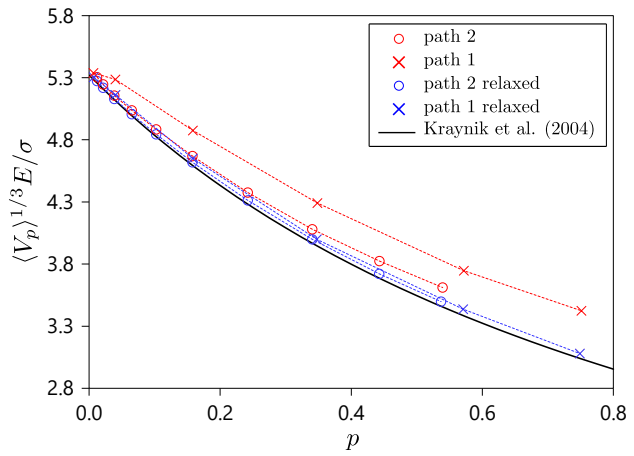

Figure 24. Surface free energy density $E$ plotted against the polydispersity parameter $p$ and compared to the theory due to Kraynik et al. [27]. $E$ is scaled by $\sigma /\left\langle V_{p}\right\rangle^{1 / 3}$, where $\sigma$ is the surface tension and $\left\langle V_{p}\right\rangle$ is the average volume of the pores.
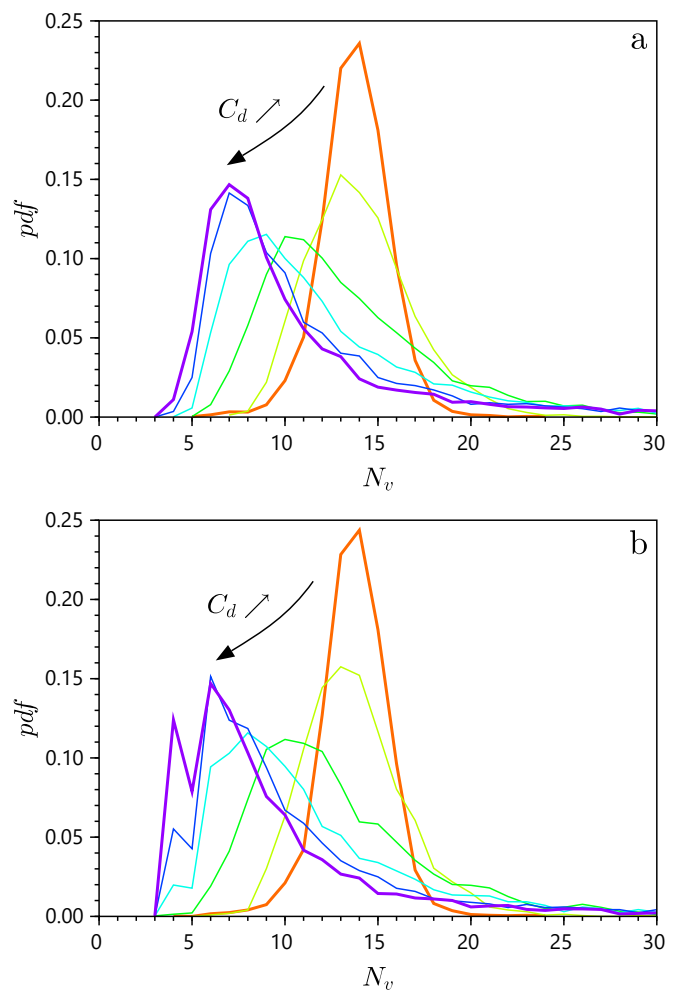

Figure 25. Distribution of the number of neighboring pores $N_{v}$ for a) Laguerre structures and b) relaxed structures (path 1). The same trends are observed with the "path 2" condition. Note that the distributions for foam samples having high polydispersity degree are not fully plotted: the number of neighbors $N_{v}$ of the largest pores of foam samples having high polydispersity degree is higher than one hundred.

of polydisperse foam structures: foam with a continuous pore-size distribution in Ref. [27, and random bidisperse foam in Ref. 28. Figure 24 shows that the energies in our relaxed foam samples follow the same relationship. These energies remain slightly higher than their theoretical values, which suggests that our relaxation pro- 

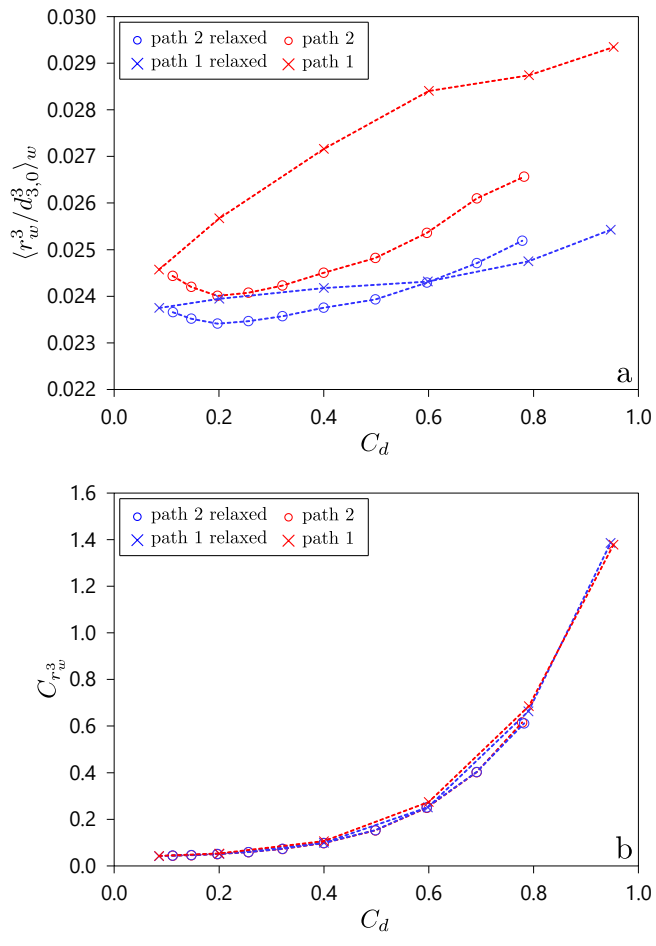

Figure 26. (a) $\left\langle r_{w}^{3}\right\rangle_{w} / d_{3,0}^{3}$ and (b) normalized standard deviation of $r_{w}^{3}$ as a function of $C_{d}$.
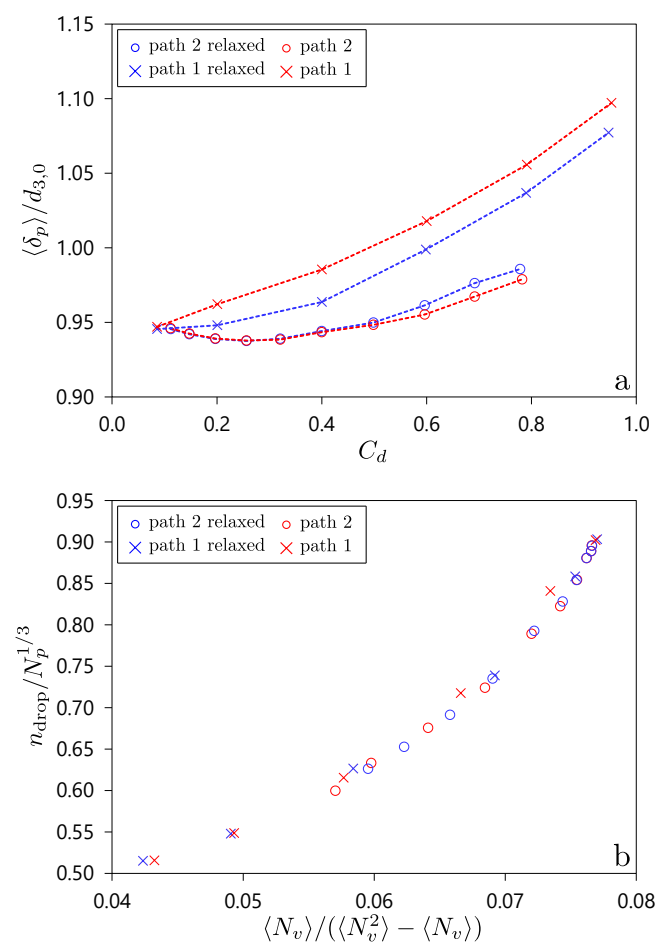

Figure 27. (a) Ratio between the mean inter-pore distance $\left\langle\delta_{p}\right\rangle$ and $d_{3,0}$ against $C_{d}$, (b) Mean number of drops $n_{\text {drop }}$ between pores and their translated counterpart as a function of the structural parameter $\left\langle N_{v}\right\rangle /\left(\left\langle N_{v}^{2}\right\rangle-\left\langle N_{v}\right\rangle\right)$.
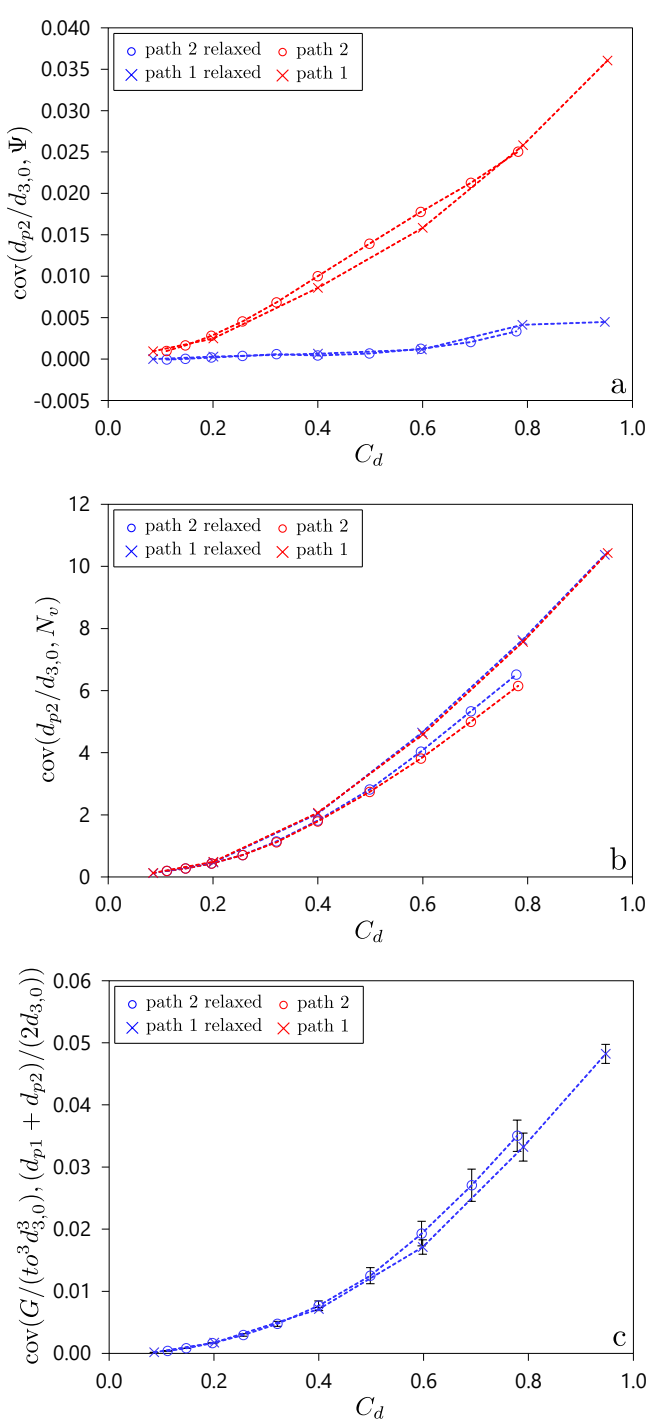

Figure 28. Effect of polydispersity degree $C_{d}$ on covariance between: (a) pore size and sphericity, (b) pore size and number of neighboring pores, (c) local conductance and the average of the pore sizes, $d_{p 1}$ and $d_{p 2}$, associated with each window (making sense in the case of constant aperture ratio). Note that the error bars gives the covariance between the local conductance and the maximal (or minimal) pore size $\max \left(d_{p 1}, d_{p 2}\right)$ (or $\left.\min \left(d_{p 1}, d_{p 2}\right)\right)$. The random variables used for the calculations of covariance are normalized or dimensionless.

cess is not as advanced as the one used by Kraynik and co-authors. However, regarding the computational costs to go further in the relaxation process, we consider our relaxed microstructures to be quite satisfactory for the purpose of our work (i.e. a deeper convergence of the relaxed microstructures would not significantly modify the relationship between the permeability and $C_{d}$, see Sec. III A). 


\section{Number of neighboring pores}

Figure 25 shows that the evolution of the distribution of $N_{v}$ with respect to the polydispersity degree is similar for both the Laguerre and relaxed structures: the distribution becomes wider and the number of neighbors associated with the maximal value decreases when the polydispersity degree increases. Similar results were found in Ref. [27].

\section{Local conductances}

Figure 26 shows the evolution of two parameters linked to the local conductances in the case of constant aperture ratio " $t_{o}$ cst" (as $\mu G_{i}=\frac{1}{3} t_{o}^{3} r_{o, i}^{3}$ ). Figure 26(a) shows that the mean local conductance is almost proportional to $d_{3,0}^{3}$. Figure 26 (b) shows that the normalized standard deviation of local conductances $C_{G}$ increases strongly as the polydispersity degree increases.

\section{Inter-pore distance}

Figure 27 shows the evolution of two parameters linked to the distance between neighbor pores. It appears that the mean inter-pore distance $\left\langle\delta_{p}\right\rangle$ and the size $d_{3,0}$ are almost equal [Fig. 27(a)]. This result is expected as $d_{3,0} \propto\left(\frac{V}{N_{p}}\right)^{1 / 3}$, and $\left(\frac{V}{N_{p}}\right)^{1 / 3}$ is currently used as the mean distance between $N_{p}$ points randomly distributed in the volume $V$. This relation is not fully observed in foam structure because pores are not just randomly distributed and have to check some constraints on pore volume (at least). Figure 27(b) shows that the structural parameter $\left\langle N_{v}\right\rangle /\left(\left\langle N_{v}^{2}\right\rangle-\left\langle N_{v}\right\rangle\right)$ and the mean number of drops $n_{\text {drop }}$ (between pores and their translated counterpart) are strongly correlated. This result shows that the structural parameter $\left\langle N_{v}\right\rangle /\left(\left\langle N_{v}^{2}\right\rangle-\left\langle N_{v}\right\rangle\right)$ seems quite powerful to characterize the pore-network structure.

\section{Covariances between microstructural parameters}

Figure 28 shows the evolution of different covariances between microstructural parameters. In Fig. 28(a), we show that the sphericity is strongly correlated to the pore size in Laguerre structures, and not in relaxed structures. These results were reported in Ref. [27. The zero covariance in relaxed structures is due to the curvatures of the interfaces leading to a constant sphericity. In Fig. 28(b), we show that the covariance between the pore size and its number of neighboring pores increases as the polydispersity degree increases (Large pores have more neighbors). And, Fig. 28. (c) shows that the covariance between local conductance and the average of the pore sizes, $d_{p 1}$ and $d_{p 2}$, associated with each window increases as the polydispersity degree increases (Large windows and high conductances are associated with large pores).
[1] J.-F. Despois and A. Mortensen, Permeability of openpore microcellular materials, Acta materialia 53, 1381 (2005).

[2] F. Chevillotte and C. Perrot, Effect of the threedimensional microstructure on the sound absorption of foams: A parametric study, The Journal of the Acoustical Society of America 142, 1130 (2017)

[3] V. Langlois, A. Kaddami, O. Pitois, and C. Perrot, Acoustics of monodisperse open-cell foam: An experimental and numerical parametric study, The Journal of the Acoustical Society of America 148, 1767 (2020).

[4] M. Hoang, G. Bonnet, H. Tuan Luu, and C. Perrot, Linear elastic properties derivation from microstructures representative of transport parameters, The Journal of the Acoustical Society of America 135, 3172 (2014).

[5] C. T. Nguyen, Acoustic foams with pore size distributions and controlled interconnections: Structures-propertiesfabrication relationships, Phd thesis Université Paris-Est Sup, France (2021).

[6] O. Doutres, N. Atalla, and K. Dong, Effect of the microstructure closed pore content on the acoustic behavior of polyurethane foams, Journal of Applied Physics 110, 064901 (2011)

[7] V. H. Trinh, V. Langlois, J. Guilleminot, C. Perrot, Y. Khidas, and O. Pitois, Tuning membrane content of sound absorbing cellular foams: Fabrication, experimen- tal evidence and multiscale numerical simulations, Materials \& Design 162, 345 (2019)

[8] D. Edouard, M. Lacroix, C. Huu, and F. Luck, Pressure drop modeling on solid foam: State-of-the art correlation, Chemical Engineering Journal 144, 299 (2008)

[9] P. Kumar and F. Topin, Predicting pressure drop in open-cell foams by adopting Forchheimer number, International Journal of Multiphase Flow 94, 123 (2017)

[10] V. Langlois, V. H. Trinh, C. Lusso, C. Perrot, X. Chateau, Y. Khidas, and O. Pitois, Permeability of solid foam: Effect of pore connections, Physical Review E 97, 053111 (2018)

[11] J. Kozeny, Ueber kapillare Leitung des Wassers im Boden, Royal Academy of Science, Vienna, Proc. Class I 136, 271 (1927).

[12] P. C. Carman, Fluid flow through granular beds, Trans. Instn Chem. Eng. 15, 150 (1937)

[13] O. Pitois, A. Kaddami, and V. Langlois, Permeability of monodisperse solid foams, Transport in Porous Media 134, 635 (2020)

[14] R. Sampson, xii. on stokes's current function, Philosophical Transactions of the Royal Society of London.(A.).

[15] I. Fatt, The Network Model in the Study of Porous Media, Ph.D. thesis, University of Southern California (1955).

[16] S. Kirkpatrick, Percolation and conduction, Reviews of modern physics 45, 574 (1973) 
[17] A. Hazen, Some physical properties of sand and gravel with special reference to their use in filtration, 24th Ann, Rep., Mass. State Board of Health, Boston, 1983 (1983).

[18] K. Terzaghi, R. Peck, and G. Mesri, Soil mechanics in engineering practice, 3rd ed., john wiley ed. (John Wiley, 1996) p. 510.

[19] B. Das, Principles of Geotechnical Engineering, cl enginee ed. (Cengage, 2009) p. 666.

[20] R. Holtz, W. Kovacs, and T. Sheahan, Introduction to Geotechnical Engineering, 2nd Edition, pearson ed. (Pearson, 2011) pp. 1-853.

[21] W. D. Carrier, Goodbye, Hazen; hello, Kozeny-Carman, Journal of Geotechnical and Geoenvironmental Engineering 129, 1054 (2003).

[22] V. Langlois, V. H. Trinh, and C. Perrot, Electrical conductivity and tortuosity of solid foam: Effect of pore connections, Physical Review E 100, 013115 (2019)

[23] D. L. Johnson, J. Koplik, and R. Dashen, Theory of dynamic permeability and tortuosity in fluid-saturated porous media, Journal of Fluid Mechanics 176, 379 (1987)

[24] M. Durand, J.-F. Sadoc, and D. Weaire, Maximum electrical conductivity of a network of uniform wires: the Lemlich law as an upper bound, Proc. R. Soc. London. Ser. A Math. Phys. Eng. Sci. 460, 1269 (2004)

[25] M. Newman, Networks: An Introduction., oxford ed. (Oxford Univ. Press, 2010) pp. 1-853.

[26] A. M. Kraynik, D. A. Reinelt, and F. van Swol, Structure of random monodisperse foam, Physical Review E 67, 031403 (2003)

[27] A. M. Kraynik, D. A. Reinelt, and F. van Swol, Structure of random foam, Physical Review Letters 93, 208301 (2004).

[28] A. M. Kraynik, D. A. Reinelt, and F. van Swol, Structure of random bidisperse foam, Colloids Surfaces A Physicochem. Eng. Asp. 263, 11 (2005).

[29] I. Vecchio, C. Redenbach, K. Schladitz, and A. M. Kraynik, Improved models of solid foams based on soap froth, Computational Materials Science 120, 60 (2016)

[30] R. Quey and L. Renversade, Optimal polyhedral description of 3D polycrystals: method and application to statistical and synchrotron x-ray diffraction data, Computer Methods in Applied Mechanics and Engineering 330, 308 (2018)

[31] K. Brakke, The surface evolver, Experimental mathematics 1, 141 (1992).

[32] A. Spettl, T. Werz, C. Krill, and V. Schmidt, Parametric representation of $3 \mathrm{D}$ grain ensembles in polycrystalline microstructures, Journal of Statistical Physics 154, 913 (2014).

[33] D. Weaire and R. Phelan, A counter-example to Kelvin's conjecture on minimal surfaces, Philos. Mag. Lett. 69,
107 (1994)

[34] I. Cantat, S. Cohen-Addad, F. Elias, F. Graner, R. Höhler, O. Pitois, F. Rouyer, A. Saint-Jalmes, R. Flatman, and S. Cox, Foams: Structure and Dynamics. (Oxford University Press, Incorporated, 2013) p. 288.

[35] A. M. Kraynik, Foam structure: from soap froth to solid foams, MRS bulletin 28, 275 (2003)

[36] D. Rowenhorst, A. Lewis, and G. Spanos, Threedimensional analysis of grain topology and interface curvature in a $\beta$-titanium alloy, Acta Materialia 58, 5511 (2010)

[37] J. Skibinski, K. Cwieka, T. Kowalkowski, B. Wysocki, T. Wejrzanowski, and K. Kurzydlowski, The influence of pore size variation on the pressure drop in open-cell foams, Materials \& Design 87, 650 (2015)

[38] D. Westhoff, J. Skibinski, O. Sedivỳ, B. Wysocki, T. Wejrzanowski, and V. Schmidt, Investigation of the relationship between morphology and permeability for open-cell foams using virtual materials testing, Materials \& Design 147, 1 (2018)

[39] S. Galam and A. Mauger, Universal formulas for percolation thresholds, Physical Review E 53, 2177 (1996).

[40] R. Cohen, K. Erez, D. ben-Avraham, and S. Havlin, Resilience of the Internet to Random Breakdowns, Phys. Rev. Lett. 85, 4626 (2000)

[41] F. Radicchi, Predicting percolation thresholds in networks, Phys. Rev. E 91, 010801(R) (2015)

[42] H. L. Frisch, J. M. Hammersley, and D. J. A. Welsh, Monte Carlo Estimates of Percolation Probabilities for Various Lattices, Phys. Rev. 126, 949 (1962)

[43] M. Li, R.-R. Liu, L. Lü, M.-B. Hu, S. Xu, and Y.-C. Zhang, Percolation on complex networks: Theory and application, Phys. Rep. 907, 1 (2021)

[44] P. Adler, J. Thovert, and V. Mourzenko, Fract. Porous Media. Vol. 9780199666 (2013) pp. 1-184.

[45] V. V. Mourzenko, J.-F. Thovert, and P. M. Adler, Macroscopic permeability of three-dimensional fracture networks with power-law size distribution, Phys. Rev. E 69, 066307 (2004)

[46] H. Fricke, A Mathematical Treatment of the Electric Conductivity and Capacity of Disperse Systems I. The Electric Conductivity of a Suspension of Homogeneous Spheroids, Phys. Rev. 24, 575 (1924).

[47] A. Ebigbo, P. Lang, A. Paluszny, and R. Zimmerman, Inclusion-Based Effective Medium Models for the Permeability of a 3D Fractured Rock Mass, Transp. Porous Media 113, 137 (2016)

[48] D. A. Klimenko, K. Hooman, and A. Y. Klimenko, Evaluating transport in irregular pore networks, Phys. Rev. E 86, 011112 (2012)

[49] P. Penfield, R. Spence, and S. Duinker, A generalized form of Tellegen's theorem, IEEE Trans. Circuit Theory 17, $302(1970)$. 